\title{
A Breathing Mode for Warped Compactifications
}

\author{
Bret Underwood \\ Department of Physics, McGill University, Montréal, QC H3A 2T8 Canada \\ bret.underwood@mcgill.ca
}

\begin{abstract}
In general warped compactifications, non-trivial backgrounds for the warp factor and the dilaton break $D$-dimensional diffeomorphism invariance, so that dilaton fluctuations can be gauged away completely and eaten by the metric. More specifically, the warped volume modulus and the dilaton are not independent, but combine into a single gauge-invariant degree of freedom in the lower dimensional effective theory, the warped breathing mode. This occurs for all strengths of the warping, even the weakly warped limit. This warped breathing mode appears as a natural zero mode deformation of backgrounds sourced by p-branes, and affects the identification of the independent degrees of freedom of flux compactifications.
\end{abstract}




\section{Introduction}

The effective theory describing the low energy limit of a compactification contains many light degrees of freedom, particularly scalar fields, arising as fluctuations of the higher dimensional fields. In string theory compactifications two such degrees of freedom are universal, appearing in any compactification: the volume modulus, a fluctuation of the metric that controls the volume of the compact space, and the dilaton, a fluctuation of the 10-dimensional scalar field that controls the strength of the string coupling. While these two degrees of freedom, and their effective theory, are most easily analyzed in the unwarped limit, many compactifications of phenomenological interest contain matter and localized objects that source non-trivial warping [1 9]. Often, the way a lower dimensional degree of freedom appears in the higher dimensional fields in the presence of warping can look very different from its relatively simple unwarped form; for some examples see [10 12]. Motivated by the simplicity and universality of the volume modulus and the dilaton, in this paper we will construct their higher-dimensional origin in warped backgrounds. We will show that in a warped background the (warped) volume modulus and the dilaton combine into a single degree of freedom, which we will call the warped breathing mode. As we will explain in more detail below, these degrees of freedom combine because of the spontaneous breaking of the higher-dimensional diffeomorphisms by the warping and the existence of non-trivial constraint equations arising from the higher-dimensional Einstein equations.

First, let us first review how fields, and their perturbations, behave on warped backgrounds. The study of dynamics and fluctuations on warped compactifications is much more complicated than the standard Kaluza-Klein (KK) reduction on direct product spaces, and has been studied extensively by a number of authors [10 30]. A number of well-known physical effects contribute to the challenges of warped effective theories:

- Wavefunctions in the internal space localize to regions of strong warping, so that terms in the effective theory involving wavefunction overlaps are more difficult to compute.

- In a KK reduction fields are expanded in a KK tower; usually only the zero mode is kept, and the higher modes integrated out, because the zero mode mass is hierarchically smaller than the KK mode masses (set by the KK scale). In spaces with regions of strong warping, however, the masses of KK modes are strongly redshifted to the IR, so that they cannot in principle be integrated out.

These effects are best understood through a simple model of a scalar field in a warped space [26]. In particular, consider the $D$-dimensional warped product of a $(p+1)$-dimensional (external) spacetime and a $(D-p-1)$-dimensional (internal) compact space, spanned by $x^{\mu}, y^{m}$ respectively, with the background metric

$$
d s_{D}^{2}=e^{2 A_{0}(y)} \hat{g}_{\mu \nu} d x^{\mu} d x^{\nu}+g_{m n}(y) d y^{m} d y^{n} .
$$

The function $A_{0}(y)$ is known as the warp factor. In addition to gravity, we will allow a $D$-dimensional scalar field $\phi$ and $D$-dimensional matter, with the action

$$
S=\frac{1}{2 \kappa_{D}^{2}} \int d^{D} x \sqrt{g_{D}}\left[R_{D}-\frac{1}{2}(\partial \phi)^{2}+\mathcal{L}_{m}\right] .
$$

We will refer to the $D$-dimensional scalar field $\phi$ as the dilaton field throughout the rest of the paper, in obvious analogy with the dilaton of 10-dimensional supergravity theories. The rest of the 
matter in $\mathcal{L}_{m}$ will contribute to generating the warped background, but fluctuations in these fields will not be important, as we will see.

Fluctuations of the dilaton on the background (1.1) take the form

$$
\phi(x, y)=\phi_{0}(y)+\delta \phi(x, y)=\phi_{0}(y)+\sum_{n} \delta \phi_{n}(x) \tilde{\phi}_{n}(y)
$$

where $\phi_{0}(y)$ is the background profile of the dilaton. Since the dilaton typically also couples to the $D$-dimensional matter generating the warp factor, we will refer to non-trivial background profiles for $A_{0}(y), \phi_{0}(y)$ as "warping" in genera 1 . In writing (1.3) we expanded the fluctuation into a tower of KK degrees of freedom $\delta \phi_{n}(x)$. The equation of motion for $\delta \phi(x, y)$, allowing for a $D$-dimensional mass $m_{\phi}^{2}$ (which can arise e.g. from fluxes, see [26]), is

$$
\begin{aligned}
\nabla_{D}^{2} \delta \phi(x, y)-m_{\phi}^{2} \delta \phi(x, y)= & \sum_{n}\left[e^{-2 A_{0}(y)} \tilde{\phi}_{n}(y) \hat{\square} \delta \phi_{n}(x)+(p+1)\left(\partial^{p} A_{0}\right)\left(\partial_{p} \tilde{\phi}_{n}(y)\right) \delta \phi_{n}(x)\right. \\
& \left.+\nabla_{D-p-1}^{2} \tilde{\phi}_{n}(y) \delta \phi_{n}(x)-m_{\phi}^{2} \tilde{\phi}_{n}(y) \delta \phi_{n}(x)\right]=0 .
\end{aligned}
$$

Writing this in terms of the $(p+1)$-dimensional mass $\hat{\square} \delta \phi_{n}(x)=m_{n}^{2} \delta \phi_{n}(x)$, we have a Schrödingertype equation for the wavefunction $\tilde{\phi}_{n}(y)$ on the compact internal space:

$$
\nabla_{D-p-1}^{2} \tilde{\phi}_{n}(y)+(p+1)\left(\partial^{p} A_{0}\right)\left(\partial_{p} \tilde{\phi}_{n}\right)+\left(e^{-2 A_{0}} m_{n}^{2}-m_{\phi}^{2}\right) \tilde{\phi}_{n}(y)=0 .
$$

The naive zero mode $\delta \phi_{0}(x) \tilde{\phi}_{0}$ is usually taken to be constant, with a $(p+1)$-dimensional mass $m_{0} \sim m_{\phi}$. This may not, however, be the lowest mass mode of the background. As discussed in [26], the wavefunction for KK modes localizes to regions of strong warping (called warped throats) where $e^{-2 A_{0}} \gg 1$. The warped throat acts like a gravitational well, redshifting the masses of the lowest KK modes to $m_{n} \sim e^{A_{0}} m_{\phi} \ll m_{0}$ so that the KK modes are much lighter than the naive zero mode. Thus, they cannot be integrated out, and must be included in the low energy effective field theory. Similar conclusions result when examining perturbations of the metric [10 12, 25].

The above warping effects arise just by examining the dilaton equation of motion, which treats the dilaton as a probe field in a fixed warped background. When the dilaton is coupled to gravity, however, additional interesting effects due to warping arise:

- Diffeomorphisms mix fields in completely different sectors (e.g. gravity and dilaton fields). Only combinations of fields from different sectors are true gauge-invariant degrees of freedom.

- Constraint equations arising from the higher dimensional equations of motion constrain fields to be dependent on each other in a non-dynamical way, reducing the overall number of independent degrees of freedom.

In particular, consider a generic fluctuation of the dilaton $\phi(x, y)=\phi_{0}(y)+\delta \phi(x, y)$. When we allow the metric to be dynamical, it is possible to transfer the entire dilaton fluctuation into the metric through a $D$-dimensional diffeomorphism $\xi^{M}(x, y)$ since

$$
\begin{aligned}
g_{M N} \rightarrow & g_{M N}-\nabla_{M} \xi_{N}-\nabla_{N} \xi_{M} ; \\
\phi(x, y) \rightarrow & \phi_{0}(y)+\delta \phi(x) \tilde{\phi}(y)+\xi^{m} \partial_{m} \phi_{0}=\phi_{0}(y) \\
& \text { with } \xi^{m}(x, y)=-\frac{\partial^{m} \phi_{0}(y)}{\left(\partial \phi_{0}\right)^{2}} \delta \phi(x, y) .
\end{aligned}
$$

\footnotetext{
${ }^{1} \mathrm{~A}$ few special cases exist, for example the string theory GKP [5] backgrounds with constant dilaton still have non-trivial metric warping.
} 
Because of the background profile $\phi_{0}(y)$, the dilaton fluctuation is no longer gauge-invariant, so it does not make sense to talk about it as a separate degree of freedom from the metric. The phenomenon arises because the background profile $\phi_{0}(y)$ spontaneously breaks the $D$-dimensional diffeomorphism invariance into a preferred slicing of $(p+1)$ - and $(D-p-1)$-dimensional spaces. In an analogous way to spontaneously broken gauge theories, the dilaton fluctuation $\delta \phi(x, y)$ can then be eaten by the metric in a suitably chosen (unitary) gauge (see [31, 32] for more discussion on the analogy with spontaneously broken gauge theories in the context of cosmology).

Next, we note that a pure dilaton fluctuation gives rise to an off-diagonal $(\mu \mathrm{m})$ component of the $D$-dimensional Einstein equations:

$$
0=\delta G_{\mu m}-\kappa_{D}^{2} \delta T_{\mu m}=-\frac{1}{2}\left(\partial_{\mu} \delta \phi(x)\right) \tilde{\phi}(y) \partial_{m} \phi_{0}(y)
$$

If no other fluctuations are turned on, this equation implies that the dilaton cannot be dynamical $\partial_{\mu} \delta \phi(x)=0$. However, inspired by our previous observation about dilaton-metric mixing through diffeomorphisms, metric fluctuations should also be included; the metric fluctuations will contribute additional terms to (1.7) so that it can be consistently solved. In particular, turning on a metric fluctuation $\delta g_{m n}=u(x) \delta_{u} g_{m n}(y)$, (1.7) becomes (schematically):

$$
\delta G_{\mu m}-\kappa_{D}^{2} \delta T_{\mu m} \sim\left(\partial_{\mu} u(x)\right) \nabla^{n} \delta_{u} g_{m n}-\frac{1}{2}\left(\partial_{\mu} \delta \phi(x)\right) \tilde{\phi}(y) \partial_{m} \phi_{0}(y)=0 .
$$

This can only be solved if the metric and dilaton degrees of freedom are identified with each other $u(x) \sim \delta \phi(x)$. More precisely, metric and dilaton fluctuations can be written in gauge invariant combinations, and these gauge invariant fluctuations are coupled through the Einstein equations.

These off-diagonal components of the Einstein equations are just the usual momentum constraint equations of the Hamiltonian formulation of general relativity (see [33]). The existence of a non-zero constraint equation is related to the fact that the dilaton fluctuation transforms under diffeomorphisms: as is typical in gauge theories, constraint equations act as the generators for gauge transformations. Thus, the two effects of gauge (diffeomorphism) non-invariance and non-zero constraint equations are just two sides of the same phenomenon.

It is important to appreciate that the mixing of the dilaton and metric degrees of freedom through the two effects just discussed is non-dynamical, in the sense that the dilaton degree of freedom $\delta \phi$ is not acting as a source for the metric degrees of freedom $\delta g$, but rather must be identified with the metric degrees of freedom. In particular, the off-diagonal Einstein equations (1.8) arise as initial value constraints on the configuration space, not as dynamical equations of motion: since they do not involve second order time derivatives, they must be imposed for all time.

Similar effects happen in $(3+1)$-dimensional cosmological backgrounds with a homogeneous time-dependent scalar field: diffeomorphisms mix the scalar field and metric perturbations, so that only gauge invariant combinations of these fields are physical, and the constraint equations couple the gauge-invariant metric and scalar field fluctuations. Pertubations can be studied using the welldeveloped formalism of cosmological perturbation theory [34,35], which emphasizes the construction of gauge-invariant variables and the role of constraints. One interesting result from this formalism is that there exists a gauge (comoving gauge) in which the scalar degree of freedom $\zeta$ is encoded as a volume rescaling factor of the spatial metric $g_{i j}=a^{2}(t) e^{2 \zeta} \delta_{i j}$.

A similar formalism can be developed for perturbations in warped backgrounds [13, 18]. The relevant metric fluctuations are the scalar components (with respect to the $(p+1)$-dimensional spacetime) of the $D$-dimensional graviton, and correspond to deformation modes of the internal space. This presents a puzzle: which deformation mode of the internal metric should the dilaton 
mix with through diffeomorphisms and the constraint equations? Fortunately, intuition from the cosmological case suggests the answer: the dilaton should mix with the "volume rescaling factor" of the internal space, otherwise known as the warped volume modulus. Mixing between a bulk scalar field and the volume modulus is warped backgrounds has been seen previously for Randall-Sundrum (RS) models [36, 37]. There, fluctuations in the bulk scalar field used in the Goldberger-Wise stabilization mechanism for the radion [38] are coupled to fluctuations in the radion itself [14-16] (for more recent investigations, see [39, 40]).

As a specific example, consider the 10-dimensional supergravity limit of string theory with fluxes and localized sources such as O-planes and D-branes, where the dilaton is the usual string theory dilaton (in 10-dimensional Einstein frame). The fluxes and localized objects act as sources for the dilaton and warp factor, so that both the dilaton and warp factor have non-trivial profiles on the internal space 2 . A priori it would seem natural to regard fluctuations of the dilaton and the volume modulus as two independent degrees of freedom. However, as argued above, and will be shown in more detail in the rest of the paper, the behavior of fluctuations under diffeomorphisms and the existence of constraint equations imply this cannot be the case: the fluctuations of the volume modulus and dilaton are controlled by a single degree of freedom, the breathing mode, which has as its 10-dimensional wavefunction a mixture of the warped volume modulus and the dilaton 3 .

In Section 2, we review the formalism of cosmological perturbation theory [34,35], and its application to perturbations on warped compactifications [13, 18]. The formalism of warped perturbation theory, namely the construction of gauge-invariant variables and the role of the constraint equations, illustrates the non-dynamical mixing between the dilaton and the metric. In the rest of the paper we will illustrate how in warped backgrounds mixing occurs between the warped volume modulus and the dilaton. In Section 3 we show how the dilaton and warped volume modulus combine through the constraint equations, and compute the kinetic term of the resulting "breathing mode" degree of freedom in the dimensionally reduced effective theory. In Section 4 we show how the breathing mode also appears naturally as a zero mode of p-brane-like compactifications. We conclude with a discussion of the implications of our results in Section 5. Appendix A contains the metric wavefunction for the warped volume modulus for arbitrary spacetime dimensions. Throughout the paper, the term "degree of freeedom" always refers to a $(p+1)$-dimensional field, while the term "field" is reserved for $D$-dimensional fields.

\section{Cosmological Perturbation Theory and Warped Com- pactifications}

It was noted in [13, 18] that there is a similarity in the structure of perturbations on cosmological and warped backgrounds. These similarities are useful for understanding the mixing of warped degrees of freedom through diffeomorphisms and constraints that we will focus on throughout the paper. In this section, we will first review cosmological perturbation theory for a scalar field on a cosmological background. We will then review the application of this formalism to warped perturbation theory for perturbations of the dilaton and the metric. In later sections, we will specialize to the case where the metric perturbations correspond to the warped volume modulus.

\footnotetext{
${ }^{2}$ Again, GKP [5] backgrounds where the dilaton is constant are an exception.

${ }^{3}$ This is different than the kinetic mixing between the dilaton and the volume modulus that arises when working in 10-dimensional string frame. This latter effect disappears after a field redefinition, which is equivalent to working in 10-dimensional Einstein frame, and preserves the number of degrees of freedom. In contrast, the mixing we are pointing out occurs even in Einstein frame, and changes the number of degrees of freedom.
} 


\subsection{Cosmological Perturbation Theory}

We will follow the general formalism for cosmological perturbation theory of [35]. Our background is a 4-dimensional FLRW spacetime on flat 3-dimensional space:

$$
d s^{2}=-d t^{2}+a^{2}(t) \delta_{i j} d x^{i} d x^{j}
$$

with scalar field matter that has a homogeneous background profile $\phi=\phi(t)$. Scalar perturbations (with respect to the spatial directions $\vec{x}$ ) about this background take the form,

$$
\begin{aligned}
d s^{2}= & -(1+2 \varphi(t, x)) d t^{2}+a^{2}(t)\left[(1-2 \psi(t, x)) \delta_{i j}+2 \partial_{i} \partial_{j} E(t, x)\right] d x^{i} d x^{j} \\
& +a(t) \partial_{i} B(t, x) d t d x^{i} \\
\phi= & \phi_{0}(t)+\delta \phi(t, x) .
\end{aligned}
$$

Altogether we have 5 scalar fluctuations $\{\varphi, \psi, E, B, \delta \phi\}$. However, not all of these fluctuations are independent. Under infinitesimal coordinate transformations $x^{\mu} \rightarrow x^{\mu}+\xi^{\mu}(t, x)$ the metric and scalar field transform as

$$
\begin{aligned}
g_{\mu \nu} & \rightarrow g_{\mu \nu}-2 \nabla_{(\mu} \xi_{\nu)} \\
\phi & \rightarrow \phi+\xi^{\mu} \partial_{\mu} \phi .
\end{aligned}
$$

In particular, spatial scalar diffeomorphisms $\xi^{\mu}=\left\{\xi^{0}(t, x), \delta^{i j} \partial_{j} \lambda(t, x)\right\}$ can be used to shuffle degrees of freedom between the metric and scalar matter sectors:

$$
\begin{aligned}
\varphi & \rightarrow \varphi-\dot{\xi}^{0} \\
\psi & \rightarrow \psi+\frac{\dot{a}}{a} \xi^{0} \\
B & \rightarrow B+\frac{1}{a} \xi^{0}-a \dot{\lambda} \\
E & \rightarrow E-\lambda \\
\delta \phi & \rightarrow \delta \phi+\dot{\phi}_{0} \xi^{0}
\end{aligned}
$$

(where a dot denotes a derivative with respect to time $t$ ). Clearly, the original scalar fluctuation variables are not gauge invariant but gauge invariant variables can be constructed [34, 35]:

$$
\begin{aligned}
\Phi_{B} & =\varphi-\frac{d}{d t}\left[a^{2}(\dot{E}-B / a)\right] \\
\Psi_{B} & =\psi+\frac{\dot{a}}{a} a^{2}(\dot{E}-B / a) \\
\delta \Phi & =\delta \phi+a^{2} \dot{\phi}_{0}(\dot{E}-B / a) .
\end{aligned}
$$

Of the 5 original scalar fluctuations, only 3 of them are gauge invariant; 2 of the scalar fluctuations are gauge artifacts, and can be removed by an appropriate gauge transformation.

Let us now examine the constraint equations arising from the Einstein equations. The standard energy and momentum constraint equations arise as the time-time and time-space components of the Einstein equations. Written in terms of the gauge invariant variables above, they are (to first order in the fluctuations):

$$
\begin{aligned}
\delta G_{00}-8 \pi G \delta T_{00} & =3 H\left(\dot{\Psi}_{B}+H \Phi_{B}\right)+\nabla^{2} \Psi_{B}+4 \pi G \delta \rho=0 \\
\delta G_{0 i}-8 \pi G \delta T_{0 i} & =2 \partial_{i}\left[\dot{\Psi}_{B}+\frac{\dot{a}}{a} \Phi_{B}-4 \pi G \dot{\phi}_{0} \delta \Phi\right]=0
\end{aligned}
$$


where $\delta \rho=\frac{1}{2} \dot{\delta} \dot{\phi} \dot{\phi}_{0}+\delta \Phi V^{\prime}\left(\phi_{0}\right)$. These equations do not contain second order time derivatives, so are not dynamical equations of motion. Instead, they are initial value constraints that must be imposed for all time. Imposing these constraint equations reduces the total number of independent degrees of freedom. As there are two independent constraint equations for our 3 gauge-invariant fields $\Phi_{B}, \Psi_{B}, \delta \Phi$, we are left with only a single independent gauge-invariant degree of freedom.

\subsection{Warped Perturbation Theory}

Inspired by the similarities between perturbations in cosmological and warped backgrounds, in this section we will develop a similar formalism for warped perturbation theory, following [13, 18].

We will take a $D$-dimensional warped product of a $(p+1)$-dimensional external spacetime and a $(D-p-1)$-dimensional internal compact space, with the background,

$$
\begin{aligned}
d s_{D}^{2} & =e^{2 A_{0}(y)} \hat{g}_{\mu \nu}(x) d x^{\mu} d x^{\nu}+e^{-2 B_{0}(y)} \tilde{g}_{m n}(y) d y^{m} d y^{n} \\
\phi & =\phi_{0}(y)
\end{aligned}
$$

Greek indices run over the external spacetime $\mu, \nu=0 \ldots p$ while lower-case latin indices run over the internal space $m, n=p+1 \ldots D-1$. We are assuming that there are other matter fields with Lagrangian $\mathcal{L}_{m}$ as well, giving rise to a background energy-momentum tensor $T_{M N}^{(0)}=\left\{T_{\mu \nu}^{(0)}, T_{m n}^{(0)}\right\}$ so that the background (2.12) is a solution to the background equations of motion. In particular, we have in mind a p-brane like background, which is discussed in more detail in Section 4. We will take the background external spacetime to be some $(p+1)$-dimensional maximally symmetric space $\hat{g}_{\mu \nu}$ such as anti-de Sitter (AdS), Minkowski, or de Sitter (dS) space. We will leave the compact internal metric $\tilde{g}_{m n}$ arbitrary, to the extent that it is a background solution; for example, the curvature of the internal space may be positive, negative, or zero, according to the solution to the background Einstein equations with the choice of matter. The warp factor $e^{-2 B_{0}(y)}$ is pulled out of the internal metric by convention, and will be chosen to be $B_{0}(y)=(p+1) /(D-p-3) A_{0}(y)$, again by convention (occasionally we will keep expressions in terms of $B_{0}$ for compactness).

Perturbations in the metric and dilaton 4 about this background that are scalar with respect to the $(p+1)$-dimensional spacetime take the form:

$$
\begin{aligned}
d s_{D}^{2}= & e^{2 A_{0}(y)}\left[(1-2 \psi(x, y)) \hat{g}_{\mu \nu}+2 \hat{\nabla}_{\mu} \partial_{\nu} E(x, y)\right] d x^{\mu} d x^{\nu}+e^{2 A_{0}(y)} \partial_{\mu} K_{m}(x, y) d x^{\mu} d y^{m} \\
& +e^{-2 B_{0}(y)}\left[\tilde{g}_{m n}(y)+2 \varphi_{m n}(x, y)\right] d y^{m} d y^{n} ; \\
\phi= & \phi_{0}(y)+\delta \phi(x, y) .
\end{aligned}
$$

As in the cosmological case, not all of these scalar fluctuations $\left\{\psi, E, K_{m}, \varphi_{m n}, \delta \phi\right\}$ are independent because of their behavior under $D$-dimensional diffeomorphisms

$$
\left(\begin{array}{l}
x^{\mu} \\
y^{m}
\end{array}\right) \rightarrow\left(\begin{array}{c}
x^{\mu}+\xi^{\mu}(x, y) \\
y^{m}+\xi^{m}(x, y)
\end{array}\right)
$$

The relevant $D$-dimensional diffeomorphisms are those which behave as scalars with respect to the $(p+1)$-dimensional spacetime: $\xi^{M}=\left\{\hat{g}^{\mu \nu} \partial_{\nu} \hat{\lambda}(x, y), \xi^{m}(x, y)\right\}$. The scalar fluctuations transform

\footnotetext{
${ }^{4}$ We will restrict perturbations in the matter sector to the dilaton in this paper. This represents a truncation of the most general set of perturbations; we leave the study of more general perturbation ansätze to future work.
} 
under these diffeomorphisms as

$$
\begin{aligned}
\varphi_{m n} & \rightarrow \varphi_{m n}-\tilde{\nabla}_{(m} \xi_{n)}+\left(\xi^{p} \partial_{p} B_{0}\right) \tilde{g}_{m n} \\
\psi & \rightarrow \psi+\xi^{m} \partial_{m} A_{0} \\
E & \rightarrow E-\hat{\lambda} \\
K_{m} & \rightarrow K_{m}-\partial_{m} \hat{\lambda}-e^{-2 B_{0}-2 A_{0}} \tilde{g}_{m n} \xi^{n} \\
\delta \phi & \rightarrow \delta \phi+\xi^{m} \partial_{m} \phi_{0}
\end{aligned}
$$

where $\hat{g}_{\mu \nu}, \tilde{g}_{m n}$ always represent the background metric. Here it is clear that the dilaton fluctuations $\delta \phi$ mix with the scalar metric fluctuations through gauge transformations due to the background profile $\phi_{0}(y)$. This can be understood in the language of spontaneously broken gauge theories: the background profile $\phi_{0}(y)$ spontaneously breaks the $D$-dimensional diffeomorphism (gauge) invariance, and the $D$-dimensional graviton (gauge field) can gain an extra degree of freedom by eating the dilaton fluctuation.

We can again define a set of gauge-invariant fields (indices on partial derivatives are raised with the warped metric $g^{m n}=e^{2 B_{0}} \tilde{g}^{m n}$ )

$$
\begin{aligned}
\Phi_{m n} & =\varphi_{m n}+e^{2 A_{0}}\left(\partial^{p} B_{0}\right)\left(K_{p}-\partial_{p} E\right) \tilde{g}_{m n}+\tilde{\nabla}_{(m}\left[e^{2 A_{0}+2 B_{0}}\left(\partial_{n)} E-K_{n)}\right)\right] \\
\Psi & =\psi+e^{2 A_{0}}\left(\partial^{p} A_{0}\right)\left(K_{p}-\partial_{p} E\right) \\
\delta \Phi & =\delta \phi+e^{2 A_{0}}\left(\partial^{p} \phi_{0}\right)\left(K_{p}-\partial_{p} E\right) .
\end{aligned}
$$

The diffeomorphism transformations have removed $(D-p)$ of the original scalar fluctuations. Eq.(2.23) shows how the scalar metric fluctuations mix with the dilaton fluctuations to create the gauge-invariant dilaton fluctuation $\delta \Phi$ when the dilaton has a non-trivial background profile.

The gauge-invariant variables also must satisfy constraints coming from the Einstein equations. For perturbations about warped backgrounds, these constraints arise from the off-diagonal $\mu \neq \nu$ and $\mu m$ Einstein equations, which read (with $\left.\Phi_{p}^{\tilde{p}} \equiv \Phi_{p q} \tilde{g}^{p q}\right)$ :

$$
\begin{aligned}
\delta G_{\mu \nu}-\left.\kappa_{D}^{2} \delta T_{\mu \nu}\right|_{\mu \neq \nu}= & \hat{\nabla}_{\mu} \partial_{\nu}\left[(p-1) \Psi-\Phi_{p}^{\tilde{p}}\right]=0 \\
\delta G_{\mu m}-\kappa_{D}^{2} \delta T_{\mu m}= & -\partial_{\mu} \partial_{m}\left[p \Psi+\Phi_{p}^{\tilde{p}}\right]+\partial_{\mu} \tilde{\nabla}_{p} \Phi_{m}^{\tilde{p}}+\partial_{\mu} \Phi_{p}^{\tilde{p}}\left[\partial_{m} A_{0}+\partial_{m} B_{0}\right] \\
& +\partial_{\mu} \Phi_{m}^{\tilde{p}}\left[(p-1) \partial_{p} A_{0}-(D-p-1) \partial_{p} B_{0}\right]+\frac{1}{2} \partial_{\mu} \delta \Phi \partial_{m} \phi_{0}=0 .
\end{aligned}
$$

Notice that the dilaton fluctuation does not contribute at linear order to the $\mu \neq \nu$ constraint equations, but it does contribute to the $\mu m$ constraint equations when the background profile is non-trivial. Thus, the gauge-invariant dilaton and metric fluctuations cannot be independent. For example, it is not possible to consider fluctuations of the dilaton by itself, since the constraint equation (2.25) is in that case

$$
\delta G_{\mu m}-\kappa_{D}^{2} \delta T_{\mu m}=-\frac{1}{2} \partial_{\mu} \delta \phi \partial_{m} \phi_{0}=0
$$

which cannot be solved for a dynamical dilaton fluctuation $\delta \phi$.

Altogether, we started with $3+(D-p-1)(D-p+2) / 2$ scalar fluctuations; after applying $2(D-p)$ constraints and gauge fixings, we are left with $1+(D-p-1)(D-p-2) / 2$ independent and gauge-invariant scalar fluctuations. Note that this is the number of independent $D$-dimensional

\footnotetext{
${ }^{5}$ See [11,25] for more discussion about the role of constraints in warped compactifications.
} 
dilaton and metric fluctuations that are scalars in $(p+1)$-dimensions, not the number of $(p+1)$ dimensional degrees of freedom. The number of $(p+1)$-dimensional degrees of freedom is in fact infinite, consisting of an infinite tower of KK-modes. In particular, the $D$-dimensional fluctuations $\left\{\Psi(x, y), \Phi_{m n}(x, y), \delta \Phi(x, y)\right\}$ can be expanded in appropriate eigenmodes of the warped internal space, e.g. $\Psi(x, y)=\sum_{n} u_{n}(x) Y^{n}(y)$ (see [11] for more discussion of eigenmodes for warped spaces), corresponding to an infinite tower of gauge-invariant, $(p+1)$-dimensional degrees of freedom.

This approach of identifying gauge-invariant variables and coupling them through the Einstein constraint equations is equivalent to the Hamiltonian construction of warped perturbation theory [25, 29]. In the Hamiltonian formalism, one performs an ADM decomposition of the metric and constructs the canonical momentum associated to the spatial metric, so that the Hamiltonian is written in terms of the canonical momentum. Invariance of the Hamiltonian under gauge transformations then enforces the constraints. While the Hamiltonian formalism is much more elegant, the slicing of spacetime into time and $(D-1)$-dimensional space obscures the physical role the background dilaton profile $\phi_{0}(y)$ plays in inducing the preferred slicing into $(p+1)$ - and $(D-p-1)$-dimensional spaces, which leads to the mixing. Since it is this latter slicing that is most important for warped backgrounds, our approach is conceptually more transparent for seeing the mixing between the dilaton and the volume modulus.

\section{Warped Breathing Mode}

In the previous section we examined the formalism of warped perturbation theory for general scalar metric and dilaton fluctuations (2.13,2.14) on the warped background (2.12). It was seen there that dilaton fluctuations generically mix non-dynamically with the metric fluctuations through the gauge-invariant fluctuations (2.21,2.23) and the constraint equation (2.25). Only when the dilaton background is completely constant do the fluctuations in the different sectors decouple.

To illustrate this more explicitly, let us now restrict ourselves to a simple subset of fluctuations to show how fluctuations from the metric and dilaton sectors combine into a single $(p+1)$-dimensional degree of freedom. We will call this degree of freedom the warped breathing mode, and it is composed of the warped volume modulus and the dilaton fluctuation, which we will consider in turn. To be clear, in this section we are considering one particular ansatz which consistently solves the constraint equations; we are not providing the most general solution for perturbations in a warped background, which is beyond the scope of this work.

\subsection{Ansatz}

Let us begin with the metric sector, and consider the warped volume modulus in a general $D$ dimensional space. This will be a generalization of the warped volume modulus for compactifications from 10 to 4 dimensions given in [12]. More details on the construction of the warped volume modulus can be found in Appendix A. As we saw in Section 2.2. the most general form of scalar metric fluctuations is given in (2.13). We will take these fluctuations to depend on a single $(p+1)$ dimensional degree of freedom $u(x)$. In the absence of dilaton fluctuations, $u(x)$ would be identified as the warped volume modulus (as seen in Appendix A). The $y$-dependent parts of the metric fluctuations will be referred to as the metric wavefunction; e.g. the fluctuations can be expanded as $\psi(x, y)=u(x) \tilde{\psi}(y), \varphi_{m n}(x, y)=u(x) \tilde{\varphi}_{m n}(y)$, etc.

In order to differentiate the warped volume modulus from other possible metric deformation modes we will require that it satisfy a few simple properties. First, the fluctuation should correspond (for some fixed gauge) to a pure trace fluctuation of the internal metric $\varphi_{m n} \propto \tilde{g}_{m n}$. This is 
motivated by the form of the unwarped volume modulus (A.3). In the warped case, however, we expect that this fluctuation may obtain some non-trivial wavefunction $\varphi_{m n}=u(x) \tilde{\varphi}(y) \tilde{g}_{m n}$ due to the background warping. Note that while it seems natural to take $\varphi_{m n}$ to be pure trace, this is not a gauge-invariant statement. The full gauge-invariant internal metric fluctuation $\Phi_{m n}(2.21)$ will not in general be pure trace, even if $\varphi_{m n}$ is. Since $\varphi_{m n}$ and $\psi$ are coupled through the constraint equations (2.24]2.25), we will also need to turn on the fluctuation $\psi=u(x) \tilde{\psi}(y)$. In general, the constraint equations also require non-zero $K_{m}, E$ fluctuations, but since $\varphi_{m n}$ is pure trace, $K_{m}$ is not sourced through the constraint equations or equations of motion, so we can set $K_{m}=0$ without loss of generality 6 .

Next, we want the breathing mode to correspond to a fluctuation in the warped volume. The relevant warped volume appears in the dimensional reduction of the $D$-dimensional Ricci scalar (we will suppress subscripts on metric determinants as $\operatorname{det} \hat{g}_{\mu \nu}=\hat{g}, \operatorname{det} \tilde{g}_{m n}=\tilde{g}$ ):

$$
\frac{1}{2 \kappa_{D}^{2}} \int \sqrt{g_{D}} R_{D} \supset \frac{1}{2 \kappa_{D}^{2}} \int \sqrt{\hat{g}} \hat{R}_{p+1} \int \sqrt{\tilde{g}} e^{-(p-1) A_{0}-(D-p-1) B_{0}}=\frac{1}{2 \kappa_{D}^{2}} \int \sqrt{\hat{g}} \hat{R}_{p+1} \tilde{V}_{W}^{(0)}
$$

where

$$
\tilde{V}_{W}^{(0)} \equiv \int \sqrt{\tilde{g}} e^{-(p-1) A_{0}-(D-p-1) B_{0}}=\int \sqrt{\tilde{g}} e^{-2 \gamma A_{0}}
$$

is the background warped volume, and $\gamma \equiv(D-2) /(D-p-3)$. Finally, we would like the metric fluctuations to reduce to the unwarped volume modulus (reviewed in Appendix A.1) in the unwarped limit.

In particular, we will take our ansatz for the warped volume modulus deformation of the metric to be:

$$
\begin{aligned}
d s^{2}= & e^{2 A(y, u(x))} e^{2 \Omega[u(x)]}\left[\hat{g}_{\mu \nu}+2 e^{(p-3) \Omega[u(x)]} \hat{\nabla}_{\mu} \partial_{\nu} u(x) E(y)\right] d x^{\mu} d x^{\nu}, \\
& +e^{-2\left(\frac{p+1}{D-p-3}\right) A(y, u(x))} \tilde{g}_{m n}(y) d y^{m} d y^{n}
\end{aligned}
$$

where we have promoted the warp factor to be a function of the warped volume modulus so that at linear order in $u(x)$

$$
A(y, u(x)) \approx A_{0}(y)+u(x) \delta A(y)+\mathcal{O}\left(u^{2}\right)
$$

for some $\delta A$ to be determined by solving the constraint equations. We have also included a Weyl factor, defined as

$$
e^{(p-1) \Omega[u(x)]}=\frac{\int \sqrt{\tilde{g}}}{\int \sqrt{\tilde{g}} e^{-2 \gamma A(y, u(x))}}=\frac{\tilde{V}_{D-p-1}}{\tilde{V}_{W}},
$$

so that the dimensionally reduced Ricci scalar is in Einstein frame, where again to linear order $\Omega[u(x)] \approx \Omega_{0}+u(x) \delta \Omega+\mathcal{O}\left(u^{2}\right)$ with $e^{(p-1) \Omega_{0}} \equiv \tilde{V}_{D-p-1} / \tilde{V}_{W}^{(0)}$. This implies $\kappa_{D}^{2}=\tilde{V}_{D-p-1} \kappa_{p+1}^{2}$, as in the unwarped case.

Comparing (3.3) to the general form for metric perturbations (2.13), we have the identifications:

$$
\begin{aligned}
\psi(x, y) & =-u(x)(\delta A(y)+\delta \Omega) ; \\
E(x, y) & =u(x) E(y) ; \\
K_{m}(x, y) & =0 ; \\
\varphi_{m n}(x, y) & =-\frac{p+1}{D-p-3} u(x) \delta A(y) \tilde{g}_{m n} .
\end{aligned}
$$
E.

${ }^{6}$ More precisely, $K_{m}=\partial_{m} K$, and there always exists a gauge where this can be shifted into the metric fluctuation 
Now we will consider the dilaton sector. As in (2.12) we will take the dilaton to have some non-trivial background profile $\phi_{0}(y)$. Let us write fluctuations of the dilaton as

$$
\phi(x, y)=\phi_{0}(y)+\delta \phi(x) \tilde{\phi}(y)
$$

where $\delta \phi(x)$ is the $(p+1)$-dimensional degree of freedom associated with the dilaton, and the dilaton fluctuation obtains a non-trivial wavefunction $\tilde{\phi}(y)$ due to the warping. We are writing the dilaton degree of freedom $\delta \phi(x)$ as a separate degree of freedom from the warped volume modulus $u(x)$, but we know that it cannot really be dynamically independent since gauge transformations can be used to shift $\delta \phi(x, y)=\delta \phi(x) \tilde{\phi}(y)$ entirely into the metric. In particular, the gauge invariant dilaton fluctuation (2.23):

$$
\delta \Phi=\delta \phi(x) \tilde{\phi}(y)-u(x) e^{2 A_{0}}\left(\partial^{p} \phi_{0}\right)\left(\partial_{p} E(y)\right)
$$

does not make sense unless $\delta \phi(x) \propto u(x)$. We can absorb the proportionality constant into the dilaton wavefunction $\tilde{\phi}(y)$ so that the degrees of freedom are identified with each other $\delta \phi(x)=u(x)$. We will call this degree of freedom, which sources both the $D$-dimensional warped volume modulus and the $D$-dimensional dilaton fluctuations, the warped breathing mode. It is clear that 3.6. 3.10) fix the gauge completely, since it is not possible to make a gauge transformation of the form (2.16-2.20) that preserves (3.6+3.10).

Even without noticing that gauge transformations mix the dilaton and warped volume modulus fluctuations together, the $(\mu m)$ constraint equation couples them in an unavoidable way:

$$
\begin{aligned}
\delta G_{\mu m}-\kappa_{D}^{2} \delta T_{\mu m}= & \left(\partial_{\mu} u(x)\right)\left(\frac{D-2}{D-p-3}\right)\left[\partial_{m} \delta A-(p+1) \delta A \partial_{m} A_{0}\right] \\
& -\frac{1}{2}\left(\partial_{\mu} \delta \phi(x)\right) \tilde{\phi}(y) \partial_{m} \phi_{0}=0 .
\end{aligned}
$$

Clearly, we cannot solve this constraint unless the warped volume modulus and dilaton fluctuations are related, so we are again led to $\delta \phi(x) \propto u(x)$. Then $\tilde{\phi}(y)$ and $\delta A(y)$ are related through this constraint. Note that it is not possible to solve this constraint equation by introducing only an off-diagonal vector metric component $K_{m} \neq \partial_{m} K$ instead 7 of a warp factor fluctuation because the resulting constraint equation is inconsistent. One way to see this is to notice that the fluctuation of the dilaton $\delta \phi(x, y)$ is a scalar with respect to the internal space, while the off-diagonal metric component $K_{m}$ is a vector. Since vectors and scalars transform differently under rotations of the internal space, it is not possible for these types of terms to cancel in the constraint equations. Alternatively, it is straightforward to compute the constraint equation including only $K_{m}$, and it is seen to be inconsistent. This conclusion about mixing between the volume modulus and dilaton fluctuations is quite general, since the mixing seen in (3.12) follows directly from the general metric ansatz (3.3) for $(p+1)$-dimensional dependent fluctuations of a $D$-dimensional scalar.

As discussed in Section 2, the dilaton fluctuation transforms under $D$-dimensional diffeomorphisms as

$$
\delta \phi(x, y) \rightarrow \delta \phi+\xi^{p} \partial_{p} \phi_{0}(y)
$$

Thus, there exists a gauge, "unitary gauge," in which the dilaton fluctuation vanishes. This can be arrived at by making a gauge transformation with gauge parameter $\xi^{p}=-u(x) \partial^{p} \phi_{0} \tilde{\phi}(y) /\left(\nabla \phi_{0}\right)^{2}$,

${ }^{7}$ Recall that a total derivative vector compensator $K_{m}=\partial_{m} K$ can always be shifted into the compensator $E(y)$ through a $(p+1)$-dimensional gauge transformation that leaves (3.12) unchanged. 
where we have made the replacement $\delta \phi(x)=u(x)$. In this gauge all of the fluctuations appear in the metric, which takes the (linearized) form:

$$
\begin{aligned}
& d s^{2}= e^{2 A_{0}(y)} e^{2 \Omega_{0}(y)}\left\{\hat{g}_{\mu \nu}\left[1+u(x)\left(\delta A+\delta \Omega+\frac{\nabla \phi_{0} \cdot \nabla A_{0}}{\left(\nabla \phi_{0}\right)^{2}} \tilde{\phi}(y)\right)\right]+2 e^{(p-3) \Omega_{0}} \hat{\nabla}_{\mu} \partial_{\nu} u(x) E(y)\right\} \\
&+e^{-2\left(\frac{p+1}{D-p-3}\right) A_{0}(y)}\left\{\tilde { g } _ { m n } \left[1-u(x)\left(\frac{p+1}{D-p-3}\right)\left(\delta A-\frac{\nabla \phi_{0} \cdot \nabla A_{0}}{\left(\nabla \phi_{0}\right)^{2}} \tilde{\phi}(y)\right]\right.\right. \\
&\left.+\tilde{\nabla}_{(m}\left(\frac{\partial_{n)} \phi_{0}}{\left(\nabla \phi_{0}\right)^{2}} \tilde{\phi}(y)\right) u(x)\right\}+\partial_{\mu} u(x) \tilde{\phi}(y) e^{-2 A_{0}(y)} \frac{\partial_{m} \phi_{0}}{\left(\nabla \phi_{0}\right)^{2}} d x^{\mu} d y^{m}
\end{aligned}
$$

Notice that in this gauge the internal metric has off-diagonal terms, and the metric is no longer block-diagonal in the internal and external directions.

In many cases of interest, the background dilaton profile $\phi_{0}(y)$ is related to the background warp factor $\phi_{0}(y)=q A_{0}(y)$, for some $q$. For example, backreaction from p-branes create warping of precisely this form, as we will explore in more detail in the next section. The breathing mode then appears in the dilaton through the ansatz:

$$
\phi(x, y)=q A(y, u(x))
$$

so that to linear order we have $\phi(x, y) \approx q A_{0}(y)+u(x) q \delta A+\mathcal{O}\left(u^{2}\right)$, e.g. $\tilde{\phi}(y)=q \delta A$. The constraint equation (3.12) can then be written in a simple form:

$$
-\left(\frac{D-2}{D-p-3}\right) \frac{e^{Q A}}{Q} \partial_{\mu} \partial_{m}\left(e^{-Q A(y, u(x))}\right)=0
$$

where $Q \equiv(p+1)+\frac{q^{2}(D-p-3)}{2(D-2)}$. Similarly as in [12], the solution is a shift ansatz for the warp factor

$$
e^{-Q A(y, u(x))}=e^{-Q A_{0}(y)}+u(x)
$$

The dilaton fluctuation does not contribute to the $\mu \neq \nu$ constraint equation (2.24) at linear order, which taking advantage of the shift form of the warp factor, takes the form

$$
\tilde{\nabla}^{2} E(y)=\frac{2 \gamma}{Q}\left[\frac{e^{-2 \gamma A(y, u(x))}}{\tilde{V}_{D-p-1}}-\frac{e^{(Q-2 \gamma) A(y, u(x))}}{\tilde{V}_{D-p-1}} \int \sqrt{\tilde{g}} e^{-2 \gamma A(y, u(x))}\right] .
$$

Notice that the left hand side of this equation is independent of the breathing mode $u(x)$, while the right hand side in general depends on $u(x)$. However, if $Q=2 \gamma(3.17)$ becomes,

$$
\tilde{\nabla}^{2} E(y)=e^{-Q A(y, u(x))}-\frac{\int \sqrt{\tilde{g}} e^{-Q A(y, u(x))}}{\tilde{V}_{D-p-1}}=e^{-2 \gamma A_{0}(y)}-\frac{\int \sqrt{\tilde{g}} e^{-2 \gamma A_{0}(y)}}{\tilde{V}_{D-p-1}},
$$

so that the right hand side only depends on the background warp factor $A_{0}(y)$ and both sides of the equation are now manifestly independent of the warped breathing mode $u(x)$. We will take this value of $Q$ from now on.

Summarizing, spacetime dependent fluctuations of the D-dimensional metric and dilaton of the 
form:

$$
\begin{aligned}
d s^{2}= & e^{2 A(y, u(x))} e^{2 \Omega[u(x)]}\left[\hat{g}_{\mu \nu}+2 e^{(p-3) \Omega} \hat{\nabla}_{\mu} \partial_{\nu} u(x) E(y)\right] d x^{\mu} d x^{\nu} \\
& +e^{-2\left(\frac{p+1}{D-p-3}\right) A(y, u(x))} \tilde{g}_{m n}(y) d y^{m} d y^{n} ; \\
\phi(x, y)= & q A(y, u(x)) \quad \text { where } \quad q^{2}=4 \frac{(D-2)^{2}}{(D-p-3)^{2}}-\frac{2(p+1)(D-2)}{D-p-3} ; \\
e^{-2 \gamma A(y, u(x))=} & e^{-2 \gamma A_{0}(y)}+u(x) ; \\
e^{(p-1) \Omega[u(x)]=} & \frac{\tilde{V}_{D-p-1}}{\int \sqrt{\tilde{g}} e^{-2 \gamma A(y, u(x))}}=\frac{1}{u(x)+\tilde{V}_{W}^{(0)} / \tilde{V}_{D-p-1}} ; \\
\tilde{\nabla}^{2} E(y)= & e^{-2 \gamma A(y, u(x))}-e^{-(p-1) \Omega[u(x)]}=e^{-2 \gamma A_{0}(y)}-\tilde{V}_{W}^{(0)} / \tilde{V}_{D-p-1},
\end{aligned}
$$

satisfy all constraint equations, and thus are a consistent ansatz for spacetime fluctuations. The warped volume modulus and the dilaton have been forced by the constraint equations to combine into a single $(p+1)$-dimensional degree of freedom, the warped breathing mode $u(x)$.

In the weakly warped limit, $e^{-2 \gamma A_{0}(y)} \approx 1$, we have $e^{(p-1) \Omega} \simeq(u(x)+1)^{-1}$ and from (3.23) the compensator vanishes to leading order $\tilde{\nabla}^{2} E \simeq 0$ so $E(y) \approx 0$. Taking $u(x)+1=e^{2(D-2) \beta /(p+1) \varphi(x)}$ with $\beta$ as in Section A.1, the metric (3.19) becomes

$$
d s^{2} \simeq e^{2 \alpha \varphi(x)} \hat{g}_{\mu \nu} d x^{\mu} d x^{\nu}+e^{2 \beta \varphi(x)} \tilde{g}_{m n} d y^{m} d y^{n}
$$

which is the metric for the unwarped volume modulus (A.3). In the completely unwarped limit $e^{-\gamma A_{0}(y)}=1$ of $(3.19,3.23)$ the unwarped volume modulus and the dilaton decouple, as they are no longer forced to be related by the off-diagonal constraint equation. In the language of constructing gauge-invariant variables, since the background profile for the dilaton is constant in the completely unwarped limit, gauge transformations no longer mix the metric and dilaton degrees of freedom. However, in the weakly warped limit the slowly varying background profile for the dilaton does still spontaneously break the $D$-dimensional gauge invariance and the metric and dilaton degrees of freedom do still mix non-dynamically, so we cannot in principle decouple these degrees of freedom. In this sense the completely unwarped limit $e^{-\gamma A_{0}(y)}=1$ of (3.19]3.23) is a singular limit - it is not smoothly connected to the weakly warped limit because the action of gauge transformations on the dilaton is not smoothly connected to the unwarped limit.

In particular, write the background warp factor as $A_{0}(y)=\epsilon f(y)$, so that $\epsilon$ controls the strength of the warping; in the large volume limit we expect $\epsilon$ to be inversely proportional to the volume. Small $\epsilon$ is the weakly warped limit, since the warp factor is then approximately constant over the internal space

$$
e^{2 A(x, y)} \approx 1-\gamma^{-1} u(x)+2 \epsilon f(y)
$$

Derivatives of the background warp factor in the internal direction are proportional to $\epsilon$ in this limit, $\partial_{m} A_{0} \sim \partial_{m} \delta A=\epsilon \partial_{m} f(y)$. The dilaton in the weakly warped limit is, from (3.14)

$$
\phi(x, y) \approx q \epsilon f(y)-\frac{q}{2 \gamma} u(x)
$$

so again derivatives of the background dilaton in the internal direction are proportional to $\epsilon$ in this limit, $\partial_{m} \phi_{0} \sim q \epsilon \partial_{m} f(y)$. From (3.12) we see that the dilaton and warped volume modulus are coupled through derivatives of the background dilaton and warp factor profiles, $\partial_{m} A_{0}, \partial_{m} \delta A, \partial_{m} \phi_{0}$. 
But since each of these terms scales with the same power of $\epsilon$, the coupling does not become parametrically small in the weakly warped, small $\epsilon$, limit. More precisely, the constraint equation becomes

$$
\left(\frac{D-2}{D-p-3}\right)\left[\epsilon \partial_{m} f(y)+\frac{(p+1)}{2 \gamma} \epsilon \partial_{m} f(y)\right]=\frac{q^{2}}{4 \gamma} \epsilon \partial_{m} f(y) .
$$

The strength of the warping $\epsilon$ completely cancels from this constraint equation, so it is independent of the size of the warping, as long as the warping is non-zero. Thus, the dilaton and warped volume modulus combine into the single warped breathing mode for all finite values of the warping, even the weakly warped limit. This has important implications for the construction of effective theories from flux compactifications, where the weakly warped limit is commonly used and the dilaton and volume modulus are assumed to be independent degrees of freedom. We will discuss this more in Section 5 .

\subsection{Dimensionally Reduced Kinetic Term}

The quadratic effective kinetic term for the warped breathing mode $u(x)$ for the system (3.19, 3.23) arises from the dimensional reduction of the $D$-dimensional Ricci scalar and dilaton action (for notational convenience, we will denote determinants of metrics as $\operatorname{det} \hat{g}_{\mu \nu}=\hat{g}$ and $\operatorname{det} \tilde{g}_{m n}=\tilde{g}$ ):

$$
\begin{aligned}
S_{e f f}^{k i n} & =\frac{1}{2 \kappa_{D}^{2}} \int \sqrt{g_{D}}\left[R_{D}-\frac{1}{2}(\partial \phi)^{2}\right]=-\frac{1}{4 \kappa_{D}^{2}} \int \sqrt{\hat{g}} \sqrt{\tilde{g}}\left[\delta g^{M N} \delta G_{M N}+\partial_{\mu} \delta \phi \partial^{\mu} \delta \phi\right] \\
& =-\frac{1}{4 \kappa_{D}^{2}} \int \sqrt{\hat{g}} \sqrt{\tilde{g}}\left[\delta g^{m n} \delta G_{m n}+\partial_{\mu} \delta \phi \partial^{\mu} \delta \phi\right]=\int \sqrt{\hat{g}}\left(\mathcal{G}_{u u}^{(g)}+\mathcal{G}_{u u}^{(\phi)}\right)\left(\partial_{\mu} u(x)\right)\left(\partial^{\hat{\mu}} u(x)\right) .
\end{aligned}
$$

In moving to the last line we used the fact that the kinetic contribution to the fluctuated external Einstein tensor $\delta G_{\mu \nu}$ vanishes once the constraints are satisfied.

We will first focus on the gravity contributions to the effective kinetic term $G_{u u}^{(g)}$. After using the equation for the wavefunction for $E(y)(3.23)$, the field space metric from gravity can be written as

$$
\begin{aligned}
\mathcal{G}_{u u}^{(g)}=\quad & -\frac{1}{4 \kappa_{D}^{2}} \int \sqrt{\tilde{g}} e^{(p-1) \Omega}\left(\frac{p+1}{D-2}\right)\left[\frac{D-2}{p-1} e^{(p-1) \Omega}+\frac{D-p-3}{2} e^{2 \gamma A}\right. \\
& \left.-\frac{D-p-3}{2} e^{(p-1) \Omega} \partial^{\tilde{m}} e^{2 \gamma A} \partial_{m} E\right] .
\end{aligned}
$$

The last term can be integrated by parts, which gives a term proportional $\tilde{\nabla}^{2} E$. Using (3.23) again the warp factor dependent pieces completely cancel out, and the numerical coefficients of the different terms miraculously combine:

$$
\mathcal{G}_{u u}^{(g)}=-\left(\frac{(p+1)^{2}(D-p-1)}{8 \kappa_{p+1}^{2}(D-2)(p-1)}\right) \frac{1}{\left(u(x)+\tilde{V}_{W}^{(0)} / \tilde{V}_{D-p-1}\right)^{2}} .
$$

The coefficient of the kinetic term (3.27) exactly matches that of the unwarped volume modulus (A.10), up to a constant shift $u \rightarrow u+\tilde{V}_{W}^{(0)} / \tilde{V}_{D-p-1}$.

The kinetic term coming from the dilaton is:

$$
\mathcal{G}_{u u}^{(\phi)}=-\frac{1}{4 \kappa_{D}^{2}} \int \sqrt{\tilde{g}} e^{-2 \gamma A} e^{(p-1) \Omega} q^{2}(\delta A)^{2}=-\frac{1}{4 \kappa_{D}^{2}} \int \sqrt{\tilde{g}} e^{(p-1) \Omega} \frac{q^{2}}{4 \gamma^{2}} e^{2 \gamma A} .
$$


Combining (3.27) and (3.28), we can write the entire effective kinetic term as,

$$
\begin{aligned}
S_{e f f}^{k i n}= & -\frac{1}{2 \kappa_{p+1}^{2}} \int \sqrt{\hat{g}} \frac{\partial_{\mu} u(x) \partial^{\hat{\mu}} u(x)}{\left(u(x)+\tilde{V}_{W}^{(0)} / \tilde{V}_{D-p-1}\right)^{2}} \times \\
& {\left[\frac{(p+1)^{2}(D-p-1)}{4(D-2)(p-1)}+\frac{q^{2}(D-p-3)}{8(D-2)^{2}} \int \sqrt{\tilde{g}} \tilde{V}_{D-p-1}^{-1} \frac{u(x)+\tilde{V}_{W}^{(0)} / \tilde{V}_{D-p-1}}{u(x)+e^{-2 \gamma A_{0}(y)}}\right] }
\end{aligned}
$$

In the weakly warped limit $e^{-2 \gamma A_{0}(y)} \approx 1$, this simplifies considerably to the form,

$$
S_{e f f}^{k i n}=-\frac{1}{2 \kappa_{p+1}^{2}} \int \sqrt{\hat{g}}\left(\frac{p}{p+1}\right) \frac{\partial_{\mu} u(x) \partial^{\hat{\mu}} u(x)}{\left(u(x)+\tilde{V}_{W}^{(0)} / \tilde{V}_{D-p-1}\right)^{2}} .
$$

\section{Breathing mode of compact p-brane solutions}

As we saw in the previous section, when the background dilaton is related to the background warp factor in a particular way (3.14) the constraints for the warped breathing mode simplify considerably. One set of examples where this happens is when the background is sourced by p-branes.

We start with the effective action for $D$-dimensional gravity, a dilaton, and a $(p+2)$-form gauge field $F_{p+2}=d C_{p+1}$ :

$$
S=\frac{1}{2 \kappa_{D}^{2}} \int d^{D} x \sqrt{g_{D}}\left[R_{D}-\frac{1}{2}(\partial \phi)^{2}-\frac{e^{-\lambda \phi}}{2(p+2) !} F_{p+2}^{2}\right]+S_{l o c},
$$

where $S_{l o c}$ denotes the action for localized sources charged under the $C_{p+1}$-field. For the usual D-branes in 10 -dimensions, $\lambda=(3-p) / 2$, but in general we will only require

$$
\lambda^{2}=4-\frac{2(p+1)(D-p-3)}{(D-2)} .
$$

The effective action (4.1) has p-brane background solutions [41-43],

$$
\begin{aligned}
d s^{2} & =H_{0}(y)^{2 a} \hat{\eta}_{\mu \nu} d x^{\mu} d x^{\nu}+H_{0}(y)^{2 b} \tilde{g}_{m n}(y) d y^{m} d y^{n} \\
e^{-\phi} & =H_{0}(y)^{\lambda / 2} \\
C_{p+1} & = \pm H_{0}(y)^{-1} \hat{\epsilon}_{p+1}
\end{aligned}
$$

where we have taken the internal space now to be Ricci flat $\tilde{R}_{m n}(\tilde{g})=0$. The exponents in the metric are defined as,

$$
a=-\frac{(D-p-3)}{2(D-2)}, \quad b=\frac{p+1}{2(D-2)},
$$

and $H_{0}(y)$ is a harmonic function on the internal space, satisfying (for localized sources)

$$
\tilde{\nabla}^{2} H_{0}(y)=\sum_{n} Q_{n} \delta^{(D-p-1)}\left(y-y_{n}\right)
$$

This background can be generalized to include additional background fluxes as well. These background fluxes act like an effective $p$-brane charge, and generalize (4.6) to include flux contributions on the right hand side as sources [44]. For example, in $D=10$ type IIB supergravity [5], additional 
3-form fluxes $G_{3}$ can behave like an effective $D 3$-brane charge and contribute additional terms to the right hand side of (4.6) as $\tilde{\nabla}^{2} H_{0}=\frac{\left|\tilde{G}_{3}\right|^{2}}{12 g_{s}}+$ localized terms. In the smeared limit, the background solutions (4.2,4.4) are just T-dual to the GKP [5] background. However, this background ansatz, and our warped breathing mode ansatz below, are more general, since they also apply in the limit of localized sources as well, where the T- duality rules do not apply. The background (4.2, 4.3) is exactly of the form proposed in the last section for the relation between the warp factor and dilaton backgrounds.

From (4.6) the equation for the harmonic function $H_{0}$ is unchanged by the shift $H \rightarrow H+u$ where $u=u(x)$ is a constant on the internal space. This shift acts like a warped volume modulus on the metric (4.2), fluctuating the warp factor. However, it also appears as a fluctuation of the dilaton and $(p+1)$-form gauge potential $C_{p+1}$. The fluctuation in $C_{p+1}$ will not play any role in determining the kinetic dynamics, e.g. it does not appear in the constraints, and will only affect the (flat) potential for $u$. However, the fluctuation of the dilaton is precisely the same as for the warped breathing mode (3.20) from Section 3. Thus, the warped breathing mode $u(x)$ from the previous section arises naturally from the shift 8 invariance $H \rightarrow H+u$ of the static $p$-brane background (4.6).

Now let us consider a general spacetime-dependent deformation of the harmonic function $H_{0}(y) \rightarrow$ $H(y, u(x))$, where the spacetime dependence in the harmonic function arises through the spacetimedependent shift $u(x)$. In order for this deformation to be a "good" deformation it must satisfy all of the constraint equations. In addition to the constraint equations coming from the Einstein equations, which we have seen before, we must also satisfy constraint equations coming from the $F_{p+2}$-form equations of motion, as well as be able to consistently solve the dilaton equation of motion. In order to solve the all of the constraint equations, our ansatz for the metric must include a $u(x)$-dependent Weyl factor on the $(p+1)$-dimensional metric and a "compensator" $E(y)$ :

$$
\begin{aligned}
d s^{2}= & H(y, u(x))^{2 a} e^{2 \Omega[u(x)]}\left[\hat{\eta}_{\mu \nu}+2 e^{(p-3) \Omega} \hat{\nabla}_{\mu} \partial_{\nu} u(x) E(y)\right] d x^{\mu} d x^{\nu} \\
& +H(y, u(x))^{2 b} \tilde{g}_{m n}(y) d y^{m} d y^{n} .
\end{aligned}
$$

To make contact with the previous section, we can rewrite (4.7) in terms of the warp factor $A(y, u(x))$ through the relation $A(y, u(x))=a \log H(y, u(x))$. The Weyl factor is

$$
e^{(p-1) \Omega}=\frac{\tilde{V}_{D-p-1}}{\int \sqrt{\tilde{g}} H(y, u(x))}=\frac{1}{u(x)+\tilde{V}_{W}^{(0)} / \tilde{V}_{D-p-1}}
$$

where $\tilde{V}_{W}^{(0)}=\int \sqrt{\tilde{g}} H_{0}(y)$ is the background warped volume. The dilaton and $C_{p+1}$-form fields also gain spacetime dependence through the harmonic function and the Weyl factor in the following way:

$$
\begin{aligned}
e^{-\phi} & =H(y, u(x))^{\lambda / 2} \\
C_{p+1} & = \pm H(y, u(x))^{-1} e^{(p+1) \Omega} \bar{\epsilon}_{p+1}
\end{aligned}
$$

where $\bar{\epsilon}_{p+1}$ is the epsilon-tensor constructed from $\bar{g}_{\mu \nu}=\hat{\eta}_{\mu \nu}+2 e^{(p-3) \Omega} \hat{\nabla}_{\mu} \partial_{\nu} u(x) E(y)$.

The dilaton and $F_{p+2}$-form field do not contribute to the $\mu \neq \nu$ constraint equation, which reads

$$
\tilde{\nabla}^{2} E(y)=H(y, u(x))-e^{-(p-1) \Omega}=H_{0}(y)-\frac{\int \sqrt{\tilde{g}} H_{0}(y)}{\int \sqrt{\tilde{g}}} .
$$

${ }^{8} \mathrm{~A}$ similar shift was also found in [45], but with a restricted form of the ansatz that limits the physical interpretation. 
Clearly (4.11) integrates to zero on both sides, and is manifestly independent of the warped breathing mode $u(x)$. As in the previous section, the dilaton does contribute to the $\mu m$ constraint equation, but the $F_{p+2}$-form field does not; in fact, $F_{p+2}$ does not contain any spacetime derivatives due to the structure of $C_{p+1}$. The $\mu m$ constraint equation then reads,

$$
\begin{aligned}
\delta G_{\mu m}-\kappa_{D}^{2} \delta T_{\mu m}= & -(p a+b(D-p-2)) \partial_{\mu} \partial_{m} \log H+(D-2) a b \partial_{\mu} \log H \partial_{m} \log H \\
& -\frac{\lambda^{2}}{8} \partial_{\mu} \log H \partial_{m} \log H=-\frac{1}{2} H^{-1} \partial_{\mu} \partial_{m} H=0 .
\end{aligned}
$$

As anticipated, solutions to (4.12) are the shift solutions,

$$
H(y, u(x))=u(x)+H_{0}(y) .
$$

However, it is not enough to just solve the Einstein constraint equations. We need to show that (4.7-4.10) are solutions to the full D-dimensional equations of motion, including the equations of motion coming from the $F_{p+2}$-form and the dilaton. The equation of motion for the $F_{p+2}$ form is:

$$
-d\left[H^{-a(p+1)+b(D-p-1)-2+\lambda^{2} / 2}\left(\tilde{\star}_{y} d_{y} H(y, u(x))\right)\right]=\sum_{n} Q_{n} \delta^{(D-p-1)}\left(y-y_{n}\right) \hat{\epsilon}_{p+1},
$$

where we denoted an exterior derivative in the internal direction as $d_{y}$. Notice that the exponent vanishes, so this simplifies to

$$
d\left[\left(\tilde{\star}_{y} d_{y} H(y, u(x))\right)\right]=\sum_{n} Q_{n} \delta^{(D-p-1)}\left(y-y_{n}\right) \tilde{\epsilon}_{D-p-1} .
$$

When the exterior derivative is in the internal direction we just find the condition that $H(y, u(x))$ must be harmonic on the internal space. Taking the exterior derivative to be in the $(p+1)$-spacetime direction, (4.15) becomes

$$
d_{x}\left[\tilde{\star}_{6} d_{y} H(y, u(x))\right]=0
$$

which is satisfied identically for the shift form (4.13) of the harmonic function. The dilaton and internal Einstein equations of motion simplify as well, reducing to

$$
\hat{\square} H(y, u(x))=0 .
$$

With the shift form of the harmonic function (4.13), this just reduces to the (linearized) equation of motion for $u(x)$ :

$$
\hat{\square} u(x)=0,
$$

which indicates that the warped breathing mode is massless.

Fluctuations of the warped breathing mode do not induce fluctuations of the p-brane itself at linear order, as can be seen by inspecting the kinetic terms of the DBI action, so (4.7 4.10) also solve the p-brane equation of motion. It is important to note that the massless warped breathing mode studied here is an independent degree of freedom from the degrees of freedom controlling the position of the p-brane in the internal space. It would be interesting to see how these degrees of freedom couple in the dimensionally reduced effective action, and we leave this for future work.

In summary, we have shown that the Einstein-dilaton-p-form system (4.1) on the background (4.2-4.4) has a $(p+1)$-dimensional "warped breathing mode" $u(x)$, realized non-trivially in the D-dimensional fields through (4.7.4.10). 


\section{Discussion}

We have argued in this paper that in generic $D$-dimensional warped compactifications to $(p+1)$ dimensions, the fluctuations associated with the warped volume modulus and the dilaton (a $D$ dimensional scalar field with a non-zero profile in the compact directions) combine into a single $(p+1)$-dimensional degree of freedom, which we have called the warped breathing mode. As discussed in Section 2, these fluctuations combine in the presence of non-trivial warping because of two effects. First, the warping breaks the $D$-dimensional diffeomorphism invariance so that the fluctuations transform non-trivially under diffeomorphisms. The gauge-invariant dilaton fluctuation (2.23) contains mixing between the metric and dilaton fluctuations. Second, the warping also leads to non-trivial constraint equations involving the fluctuations arising from the $D$-dimensional Einstein equations. We explicitly illustrated this in Section 3 by constructing the $D$-dimensional wavefunction for the warped volume modulus and the dilaton, and showed that solving the Einstein constraint equations forces these fluctuations to combine into a single degree of freedom. In Section 4 we showed that the warped breathing mode is the natural zero mode on the warped backgrounds sourced by p-branes, indicating that it is indeed the correct low-energy degree of freedom in the presence of objects that source warping.

The mechanisms of mixing discussed here (spontaneous breaking of diffeomorphisms and nontrivial constraint equations) are not restricted to a bulk scalar field and volume modulus. P-form gauge fields that obtain a non-zero background profile will lead to similar mixings between the p-form and metric degrees of freedom (see [10]). Further, gauge transformations associated with the p-form fields themselves can also mix degrees of freedom in different sectors when the p-form fluxes have non-trivial backgrounds. For example, in type IIB supergravity, Chern-Simons couplings between the 4-form and 2-form gauge potentials $C_{4}, C_{2}, B_{2}$ implies that $C_{4}$ transforms under gauge transformations of the 2-form potentials when the 2-forms have background 3-form field strengths, e.g.:

$$
\begin{aligned}
& C_{2} \rightarrow C_{2}+d \zeta_{1}^{C} \\
& B_{2} \rightarrow B_{2}+d \zeta_{1}^{B} \\
& C_{4} \rightarrow C_{4}+\frac{1}{2} \zeta_{1}^{C} \wedge H_{3}^{(0)}+\frac{1}{2} \zeta_{1}^{B} \wedge F_{3}^{(0)}
\end{aligned}
$$

Generalizing the gauge-invariance and constraint equation arguments given here, fluctuations in $C_{4}$ will mix with those of $B_{2}, C_{2}$; this was seen explicitly in [6, 12, for the axion of $C_{4}$ in GKP [5] backgrounds. Similar mixing effects of p-form fluctuations will likely arise in the "generalized BPS-like" backgrounds of [44] or [46]. Thus, the approach given here of identifying the gaugeinvariant combinations of fluctuations that are independent under the constraint equations is a useful organizing scheme for understanding the structure of effective theories arising from compactification in general.

The effects discussed in this paper all arise for non-trivial warping, where the background profiles are non-constant $\partial_{m} A_{0}(y), \partial_{0} \phi_{0}(y) \neq 0$. In the weakly warped limit the background profiles approach a constant e.g. $e^{\phi_{0}(y)}, e^{2 A_{0}(y)} \sim 1+2 \epsilon f(y)$ for some small $\epsilon$, where $\epsilon$ is inversely proportional to some power of the volume. Thus, it seems we can sidestep the subtleties associated with warping as long as we are willing to work at a sufficiently large volume where there are no strongly warped regions. This line of argument certainly works to remove the problems due to warping of wavefunction localization and integrating out KK modes discussed in the introduction. In the large volume limit, the gravitational potential well generated by the warping disappears, so that wavefunctions spread out over the entire internal space. Likewise, KK modes become hierarchically 
more massive than the zero mode in the large volume limit (see [10, 23, 26] for more discussion of these effects).

But it is hard to see how the non-dynamical mixing from the diffeomorphisms and constraint equations can be removed by a large volume limit: in the completely unwarped limit the mixing between the dilaton and the volume modulus vanishes. At strong warping, however, the dilaton and volume modulus combine into a single degree of freedom. At the level of the equations, we found in Section 3 that the constraint equation for the warped volume modulus $u(x)$ and the dilaton $\delta \phi(x)$ takes the (schematic) form:

$$
\delta G_{\mu m}-\kappa_{D}^{2} \delta T_{\mu m}=0 \Rightarrow\left(\partial_{\mu} u(x)\right) \partial_{m} A_{0}(y) \sim\left(\partial_{\mu} \delta \phi(x)\right) \partial_{m} \phi_{0}(y)
$$

where the background profiles scale in the same way in the weakly warped limit $\partial_{m} A_{0}, \partial_{m} \phi_{0} \sim$ $\epsilon \partial_{m} f(y)$. The scaling with the strength of the warping $\epsilon$ cancels out but the non-zero derivative of the profile does not. The only way to solve this equation for any finite strength of the warping $\epsilon$, then, is if the dilaton and the warped volume modulus combine into a single breathing mode, even in the weakly warped limit.

Note that we have not solved the full set of linearized equations for a general set of perturbations on the most general background, so additional independent degrees of freedom may be present. In particular, there should be KK modes of the warped breathing mode, but it is not at all clear what form such fluctuations will take. We have not attempted to study such perturbations. Instead, however, we have shown that the dilaton cannot be taken to be independent from the warped volume modulus in a warped background, as is commonly done. We leave the study of more general perturbation ansätze to future work.

\section{Acknowledgments}

We would like to thank R. Brandenberger, A. Castro, K. Dasgupta, D. George, D. Marsh, L. McAllister, G. Shiu, and Y. Wang for useful related discussions and comments, and would particularly like to thank A. Frey and T. Van Riet for comments on an earlier version of the paper. B.U. is supported in part by NSERC, an IPP (Institute of Particle Physics, Canada) Postdoctoral Fellowship, and by a Lorne Trottier Fellowship at McGill University.

\section{A Warped Volume Modulus}

In unwarped backgrounds the volume modulus is easy to identify: it is just a simple rescaling of the internal metric (together with a Weyl rescaling of the spacetime metric so that the lower dimensional spacetime is in Einstein frame). In warped backgrounds, the definition of the warped volume modulus is not as simple [10,12]. In [12] the warped volume modulus was constructed for warped compactifications from 10 to 4 dimensions, and it was seen there that the volume modulus mixes with the warp factor and gives rise to additional "compensator" terms in the metric. In this Appendix we generalize the construction of [12] to warped compactifications with arbitrary numbers of dimensions, seeing again that the warped volume modulus mixes with the warp factor and gives rise to metric compensators. In the unwarped limit these mixings and compensators vanish, so that the fluctuation reverts back to a simple rescaling of the internal metric.

The mode we would like to study is the warped generalization of the volume modulus. In order to differentiate the warped volume modulus from other possible deformation modes, we will require that it satisfies a few simple properties: the fluctuation should correspond, in some gauge, to a 
pure trace fluctuation $\delta \varphi_{m n} \sim \tilde{g}_{m n}$; it should correspond to a fluctuation in the "warped volume" $\tilde{V}_{W}^{(0)}=\int \sqrt{\tilde{g}} e^{(p-1) A_{0}-(D-p-1) B_{0}}$; it should satisfy all of the constraints; and it should reduce to the unwarped volume modulus in the unwarped limit. We will first review the unwarped volume modulus, then construct an ansatz for the warped volume modulus that meets the above criteria.

We are assuming that there is some bulk matter with energy-momentum tensor $T_{M N}$, such that the background metric is a solution to the background Einstein equations $G_{M N}-\kappa_{D}^{2} T_{M N}=0$ for a maximally symmetric spacetime metric $\hat{g}_{\mu \nu}$ and arbitrary internal space $\tilde{g}_{m n}$. We are taking the background matter fields (including the dilaton) to be fixed with no fluctuations, so they are only important for sourcing the background, and we will not need their detailed form.

\section{A.1 Review: Unwarped Volume Modulus}

Let us first start by reviewing the unwarped volume modulus, following the notation of [47]. The unwarped metric corresponds to constant warp factors, which we will set to unity by rescaling the $x^{\mu}, y^{m}$ coordinates:

$$
d s_{D}^{2}=\hat{g}_{\mu \nu}(x) d x^{\mu} d x^{\nu}+\tilde{g}_{m n}(y) d y^{m} d y^{n} .
$$

A fluctuation of the volume modulus $\varphi$ corresponds to a fluctuation of the overall scale of the internal metric,

$$
d s_{D}^{2}=\hat{g}_{\mu \nu}(x) d x^{\mu} d x^{\nu}+e^{2 \beta \varphi(x)} \tilde{g}_{m n}(y) d y^{m} d y^{n},
$$

as can be seen by the fact that the internal volume scales with $\varphi$ as $V_{D-p-1}=e^{-(p-1) \varphi} \int \sqrt{\tilde{g}}$. However, in order to remain in $(p+1)$-dimensional Einstein frame after compactification, we must also include a modulus-dependent Weyl rescaling of the $(p+1)$-dimensional spacetime,

$$
d s_{D}^{2}=e^{2 \alpha \varphi(x)} \hat{g}_{\mu \nu}(x) d x^{\mu} d x^{\nu}+e^{2 \beta \varphi(x)} \tilde{g}_{m n}(y) d y^{m} d y^{n},
$$

where $\beta=-\frac{(p-1)}{D-p-1} \alpha$ ensures the Einstein frame condition. This also leads to the identification of the lower dimensional Newton's constant $\kappa_{D}^{2}=\tilde{V}_{D-p-1} \kappa_{p+1}^{2}$, where $\tilde{V}_{D-p-1}=\int \sqrt{\tilde{g}_{D-p-1}}$ is the $(D-p-1)$-dimensional (unwarped) volume. As we will see soon, if we further choose

$$
\alpha^{2}=\frac{D-p-1}{2(D-2)(p-1)}
$$

then $\varphi$ will be a canonically normalized scalar field in the resulting $(p+1)$-dimensional effective theory. For small spacetime fluctuations of the volume modulus:

$$
\varphi(x)=\varphi_{0}+\delta \varphi(x)+\ldots
$$

the fluctuation (A.3) in the notation of warped perturbation theory from Section 2.2 corresponds to the gauge-invariant metric fluctuations,

$$
\begin{aligned}
\Phi_{m n}(x, y) & =\beta \delta \varphi(x) \tilde{g}_{m n}(y) \\
\Psi(x, y) & =-\alpha \delta \varphi(x) .
\end{aligned}
$$

The constraint equations $\left.\delta G_{\mu \nu}\right|_{\mu \neq \nu}, \delta G_{\mu m}$ are satisfied identically for the ansatz (A.3), and the part of the internal Einstein equation proportional to the kinetic term is:

$$
G_{m n}=-e^{2(\beta-\alpha) \varphi_{0}} \tilde{g}_{m n} \hat{\square} \delta \varphi \frac{\alpha(D-2)}{D-p-1}+\ldots
$$


where by ... we mean that only the kinetic pieces are shown. We can construct the $(p+1)$ dimensional (quadratic) effective kinetic term for the volume modulus $\varphi$ by reducing the Ricci scalar, which becomes:

$$
\begin{aligned}
S_{e f f} & =\frac{1}{2 \kappa_{D}^{2}} \int \sqrt{g_{D}} R_{D}=\frac{1}{4 \kappa_{D}^{2}} \int \sqrt{g_{D}} \delta G_{M N} \delta g^{M N} \\
& =\frac{1}{4 \kappa_{D}^{2}} \int \sqrt{g_{D}} \delta G_{m n} \delta g^{m n}=-\int \sqrt{\hat{g}_{p+1}} \frac{\left(\partial_{\mu} \delta \varphi\right)\left(\partial^{\hat{\mu}} \delta \varphi\right)}{4 \kappa_{p+1}^{2}}
\end{aligned}
$$

where in the last step we used the definition of $\alpha$ above (A.4 so that $\varphi$ is canonically normalized (the extra factor of $\left(2 \kappa_{p+1}\right)^{-1}$ is a common convention). Another convenient parameterization of the volume modulus is in terms of the "breathing mode" $u(x)=e^{2(D-2) \beta /(p+1) \varphi(x)}$ for which the metric and effective kinetic term become:

$$
\begin{aligned}
d s_{D}^{2} & =u(x)^{-\frac{(p+1)(D-p-1)}{(D-2)(p-1)}} \hat{g}_{\mu \nu} d x^{\mu} d x^{\nu}+u(x)^{\frac{p+1}{D-2}} \tilde{g}_{m n} d y^{m} d y^{n} \\
S_{e f f} & =-\int \sqrt{\hat{g}_{p+1}}\left(\frac{(p+1)^{2}(D-p-1)}{8 \kappa_{p+1}^{2}(D-2)(p-1)}\right) \frac{\left(\partial_{\mu} u(x)\right)\left(\partial^{\hat{\mu}} u(x)\right)}{u(x)^{2}}
\end{aligned}
$$

\section{A.2 Warped Volume Modulus}

As discussed at the beginning of this section, we would like to construct an ansatz for the warped volume modulus on the warped background

$$
d s^{2}=e^{2 A_{0}(y)} \hat{g}_{\mu \nu}(x) d x^{\mu} d x^{\nu}+e^{-2 B_{0}(y)} \tilde{g}_{m n}(y) d y^{m} d y^{n},
$$

where we will take $B_{0}(y)=(p+1) /(D-p-3) A_{0}(y)$ as in the main text. The relevant "warped volume" appears in the dimensional reduction of the $D$-dimensional Ricci scalar (we will suppress subscripts on metric determinants as $\left.\operatorname{det} \hat{g}_{\mu \nu}=\hat{g}, \operatorname{det} \tilde{g}_{m n}=\tilde{g}\right)$ :

$$
\frac{1}{2 \kappa_{D}^{2}} \int \sqrt{g_{D}} R_{D} \supset \frac{1}{2 \kappa_{D}^{2}} \int \sqrt{\hat{g}} \hat{R}_{p+1} \int \sqrt{\tilde{g}} e^{-(p-1) A_{0}-(D-p-1) B_{0}}=\frac{1}{2 \kappa_{D}^{2}} \int \sqrt{\hat{g}} \hat{R}_{p+1} \tilde{V}_{W}^{(0)}
$$

where

$$
\tilde{V}_{W}^{(0)} \equiv \int \sqrt{\tilde{g}} e^{-(p-1) A_{0}-(D-p-1) B_{0}}=\int \sqrt{\tilde{g}} e^{-2 \gamma A_{0}(y)}
$$

is the warped volume, with $\gamma \equiv(D-2) /(D-p-3)$.

The ansatz for the warped volume modulus we will use i\&9:

$$
\begin{aligned}
d s^{2}= & e^{2 A(y, u(x))} e^{2 \Omega[u(x)]}\left[\hat{g}_{\mu \nu}+2 e^{(p-3) \Omega[u(x)]} \hat{\nabla}_{\mu} \partial_{\nu} u(x) E(y)\right] d x^{\mu} d x^{\nu} \\
& +e^{-2\left(\frac{p+1}{D-p-3}\right) A(y, u(x))} \tilde{g}_{m n}(y) d y^{m} d y^{n}
\end{aligned}
$$

where we have promoted the warp factor to be a function of the warped volume modulus so that at linear order in $u(x)$

$$
A(y, u(x)) \approx A_{0}(y)+u(x) \delta A(y)+\mathcal{O}\left(u^{2}\right)
$$

\footnotetext{
${ }^{9} \mathrm{~A}$ qualitative argument for this form is given in the beginning of Section 3.1
} 
We have also included a Weyl factor, defined as

$$
e^{(p-1) \Omega}=\frac{\int \sqrt{\tilde{g}}}{\int \sqrt{\tilde{g}} e^{(p-1) A} e^{-(D-p-1) B}}=\frac{\tilde{V}_{D-p-1}}{\tilde{V}_{D-p-1}^{W}}
$$

so that the dimensionally reduced Ricci scalar is in Einstein frame, where again to linear order $\Omega[u(x)] \approx \Omega_{0}+u(x) \delta \Omega+\mathcal{O}\left(u^{2}\right)$ with $e^{(p-1) \Omega_{0}} \equiv \tilde{V}_{D-p-1} / \tilde{V}_{W}^{(0)}$. This implies $\kappa_{D}^{2}=\tilde{V}_{D-p-1} \kappa_{p+1}^{2}$, as in the unwarped case. For convenience, we will denote the unwarped metric with the compensator piece as

$$
\bar{g}_{\mu \nu}=\hat{g}_{\mu \nu}+2 e^{(p-3) \Omega} \hat{\nabla}_{\mu} \partial_{\nu} u(x) K(y) .
$$

The constraint equations come from the off-diagonal parts of the $D$-dimensional Einstein equations, and are:

$$
\begin{aligned}
&\left.\delta G_{\mu \nu}\right|_{\mu \neq \nu}=\left(\hat{\nabla}_{\mu} \partial_{\nu} u\right)\left\{e^{2 A+2 B+(p-1) \Omega\left[\tilde{\nabla}^{2} E\right.}\right.\left.+(D-p-3) \partial_{m} B \partial^{\tilde{m}} E-(p+1) \partial_{m} A \partial^{\tilde{m}} E\right] \\
&-(1-p)(\delta \Omega+\delta A)+(D-p-1) \delta B\}=0 ; \\
& \delta G_{\mu m}=-p \partial_{\mu} \partial_{m} A+(D-p-2) \partial_{\mu} \partial_{m} B-(D-2) \partial_{\mu} B \partial_{m} A=0 .
\end{aligned}
$$

In the absence of dilaton fluctuations, the $(\mu m)$ constraint equation (A.18) becomes,

$$
\delta G_{\mu m}=-\left(\frac{D-2}{D-p-3}\right) \frac{e^{(p+1) A}}{(p+1)} \partial_{\mu} \partial_{m}\left(e^{-(p+1) A}\right)=0,
$$

which is solved by the generalized shift ansatz for the warp factor,

$$
e^{-(p+1) A(y, u(x))}=e^{-(p+1) A_{0}(y)}+u(x)
$$

where $A_{0}(y)$ is a background warp factor. The Weyl factor becomes,

$$
e^{(p-1) \Omega}=\frac{\tilde{V}_{D-p-1}}{\int \sqrt{\tilde{g}} e^{-2 \gamma A(x, y)}} .
$$

The constraint equation (A.17) is solved by:

$$
\begin{aligned}
\tilde{\nabla}^{2} E & =e^{-2 \gamma A}[2 \gamma \delta A+(1-p) \delta \Omega] e^{-(p-1) \Omega} \\
& =\frac{2 \gamma}{p+1}\left[\frac{e^{-2 \gamma A}}{\tilde{V}_{D-p-1}} \int \sqrt{\tilde{g}} e^{(-2 \gamma+p+1) A}-\frac{e^{(-2 \gamma+p+1) A}}{\tilde{V}_{D-p-1}} \int \sqrt{\tilde{g}} e^{-2 \gamma A}\right] .
\end{aligned}
$$

Summarizing, we have shown that the ansatz,

$$
\begin{aligned}
d s^{2}=\quad & \left(e^{-(p+1) A_{0}(y)}+u(x)\right)^{-2 /(p+1)} e^{2 \Omega[u(x)]}\left[\hat{g}_{\mu \nu}+2 \hat{\nabla}_{\mu} \partial_{\nu} u(x) E(y)\right] d x^{\mu} d x^{\nu} \\
& +\left(e^{-(p+1) A_{0}(y)}+u(x)\right)^{2 /(D-p-3)} \tilde{g}_{m n} d y^{m} d y^{n}
\end{aligned}
$$

with $E(y)$ solving (A.23) and $\Omega$ defined by (A.21), solves the linearized warped constraint equations. It should be straightforward to generalize this to the non-linear level as in [12. Clearly, if we turn off the volume modulus fluctuation $u(x)=0$, we return to the background (A.11). In the unwarped limit, $e^{(p-1) A_{0}(y)} \rightarrow 1$, with the identification $u(x)+1=e^{(D-p-3) \beta \varphi(x)}$ the metric (A.24) becomes the ansatz for the unwarped volume modulus (A.3) as in Section A.1. This ansatz for the metric fluctuations meets our criteria for the warped volume modulus outlined at the beginning of this section: the wavefunction has a pure trace component, it corresponds to a fluctuation of the warped volume $\tilde{V}_{W}$, it solves the constraint equations, and it reduces to the unwarped volume modulus in the unwarped limit. 


\section{A.2.1 Kinetic Term}

The effective kinetic term for $u(x)$ can be obtained from a dimensional reduction of the $D$-dimensional Ricci scalar:

$$
\begin{aligned}
S_{e f f} & =\frac{1}{2 \kappa_{D}^{2}} \int \sqrt{g_{D}} R_{D}=-\frac{1}{4 \kappa_{D}^{2}} \int \sqrt{\hat{g}} \sqrt{\tilde{g}} \delta g^{M N} \delta G_{M N} \\
& =-\frac{1}{4 \kappa_{D}^{2}} \int \sqrt{\hat{g}} \sqrt{\tilde{g}} \delta g^{m n} \delta G_{m n} .
\end{aligned}
$$

In the last step we used the fact that the kinetic contribution to the fluctuated external Einstein tensor $\delta G_{\mu \nu}$ vanishes once the constraints are satisfied. The kinetic part of the Einstein equation in the internal directions is

$$
\begin{aligned}
\delta G_{m n}= & \hat{\square} u(x)\left[\tilde{g}_{m n} e^{-2 A-2 B-2 \Omega}\{p(\delta A+\delta \Omega)-(D-p-2) \delta B\}\right. \\
& +e^{(p-3) \Omega}\left[-\tilde{\nabla}_{m} \partial_{n} K-\left(\partial_{m}(A+B) \partial_{n} E+\partial_{m} E \partial_{n}(A+B)-\tilde{g}_{m n} \partial_{p} E \partial^{\tilde{p}} B\right)\right. \\
& \left.\left.+\tilde{g}_{m n} \tilde{\nabla}^{2} E+\partial_{p} A \partial^{\tilde{p}} E \tilde{g}_{m n}+2\left\{(D-p-3) \partial_{p} B \partial^{\tilde{p}} E-(p+1) \partial_{p} A \partial^{\tilde{p}} E\right\}\right]\right]+\ldots
\end{aligned}
$$

The fluctuated internal metric is:

$$
\delta g_{m n}=-2 \delta B g_{m n}, \quad \delta g^{m n}=g^{m p} g^{n q} \delta g_{p q}=-2 \delta B g^{m n}=-2 \delta B e^{2 B} \tilde{g}^{m n} .
$$

Using (A.22) we can write $\delta g^{m n} \delta G_{m n}$ as:

$$
\delta g^{m n} \delta G_{m n} \quad=-2 u \hat{\square} u \delta B e^{-2 A-2 \Omega}\left[(D-2)(\delta \Omega+\delta A)+\gamma(D-p-3) e^{2 \gamma A+2 \Omega} \partial^{\tilde{m}} A \partial_{m} E\right] .
$$

Writing the effective kinetic term as:

$$
-\frac{1}{4 \kappa_{D}^{2}} \int \sqrt{g_{D}} \delta g^{m n} \delta G_{m n}=\int \sqrt{\hat{g}} G_{u u} u \hat{\square} u
$$

the field space metric is:

$$
G_{u u}=\frac{1}{4 \kappa_{D}^{2}} \int \sqrt{\tilde{g}} \frac{2(D-2)}{D-p-3} e^{(p-1) \Omega} e^{(p+1-2 \gamma) A}\left[\delta \Omega+\delta A+e^{2 \gamma A+2 \Omega} \partial^{\tilde{m}} A \partial_{m} E\right] .
$$

The last term can be integrated by parts, leaving us with

$$
\begin{aligned}
G_{u u}=-\frac{1}{2 \kappa_{p+1}^{2}} \frac{D-2}{(p+1)(D-p-3)} & {\left[\frac{4 p \gamma}{(p-1)(p+1)} e^{2(p-1) \Omega} \frac{\left(\tilde{V}_{W}^{(p+1-2 \gamma)}\right)^{2}}{\tilde{V}_{D-p-1}}\right.} \\
& \left.+\left(1-\frac{2 \gamma}{p+1}\right) e^{(p-1) \Omega} \frac{V_{W}^{(2(p+1)-2 \gamma)}}{\tilde{V}_{D-p-1}}\right],
\end{aligned}
$$

where we are using the notation $\tilde{V}_{W}^{\alpha} \equiv \int \sqrt{\tilde{g}} e^{\alpha A_{0}(y)}$. 
To make contact with the unwarped case it is also convenient to change variables to $u(x)=$ $e^{-(D-p-3) \beta \varphi(x)}$, with corresponding effective kinetic term,

$$
\begin{aligned}
S_{\text {eff }}= & \int \sqrt{\hat{g}} G_{\varphi \varphi} \varphi \hat{\square} \varphi ; \\
G_{\varphi \varphi}= & -\frac{1}{2 \kappa_{p+1}^{2}} \frac{(p-1)}{(D-p-1)(p+1)^{2}} e^{-2(D-p-3) \beta \varphi}\left[\frac{4 p(D-2)}{(p-1)} e^{2(p-1) \Omega} \frac{\left(\tilde{V}_{W}^{(p+1-2 \gamma)}\right)^{2}}{\tilde{V}_{D-p-1}}\right. \\
& \left.+\left(D(p-1)-p^{2}-4 p+1\right) e^{(p-1) \Omega} \frac{V_{W}^{(2(p+1)-2 \gamma)}}{\tilde{V}_{D-p-1}}\right] .
\end{aligned}
$$

In the unwarped limit, the volume factors all cancel out, and remarkably all of the factors of $D$ and $p$ in (A.33) cancel as well, leading to the canonically normalized kinetic term as in the previous section. However, for non-trivial warping we see that now the kinetic term for the "traditional"

volume modulus $\varphi$ is no longer canonically normalized in general. Of course, it is straightforward to canonically normalize the kinetic term by an appropriate field redefinition. The point here is that the warped volume modulus does not automatically have the same kinetic term as the unwarped volume modulus - they are related by a field redefinition. This is in contrast to that found in [12], where the kinetic terms for the warped and unwarped volume moduli were found to be identical, a fact crucial for verifying the conjectured $\mathcal{N}=1$ Kähler potential for the volume modulus.

\section{A.3 Special Cases}

An interesting set of special cases of the warped volume modulus emerges when $p+1=2 \gamma$. In these cases, the second term in (A.31) vanishes, and the first term simplifies considerably, so that the field space metric becomes:

$$
G_{u u}=-\frac{1}{2 \kappa_{p+1}^{2}}\left(\frac{p}{p+1}\right) \frac{1}{\left(u(x)+\tilde{V}_{W}^{(0)} / \tilde{V}\right)^{2}} .
$$

Taking the maximal spacetime dimension to be $D=11$, the only integer values of $D$ and $p$ for which this is satisfied are:

$$
\begin{array}{lll}
p=2, & D=11: & D=11 \rightarrow 2+1(M 2) \\
p=3, & D=10: & D=10 \rightarrow 3+1(D 3) \\
p=5, & D=10: & D=11 \rightarrow 5+1(M 5) .
\end{array}
$$

These special cases are precisely those for which the warped product structure of the volume modulus deformed metric (A.14) matches that of the corresponding $p$-brane solutions without a dilaton: D3branes in 10-dimensional supergravity, and, more trivially, $M 2$ - and $M 5$-branes in 11-dimensional supergravity.

In particular, (A.14) for the case of $D=11$ reduced to $p+1=3$-dimensional Minkowski space has the metric (relabeling $2 A(y, u(x))=-\phi(y, u(x))$ by convention):

$$
\begin{aligned}
d s_{11}^{2}= & e^{-\phi(y, u(x))} e^{2 \Omega(u(x))}\left[\hat{\eta}_{\mu \nu}+2 e^{-\Omega} \partial_{\mu} \partial_{\nu} u(x) K(y)\right] d x^{\mu} d x^{\nu} \\
& +e^{\frac{1}{2} \phi(y, u(x))} \tilde{g}_{m n}(y) d y^{m} d y^{n}
\end{aligned}
$$


with the shift ansatz (A.20) taking the form,

$$
e^{\frac{3}{2} \phi(y, u(x))}=e^{\frac{3}{2} \phi_{0}(y)}+u(x) .
$$

The warp factor structure in (A.35) is exactly the same as the supergravity solution corresponding to an extremal M2-brane [48]. Upon supersymmetric compactification to $(2+1)$-dimensional Minkowski space on spacetime filling $M 2$-branes, supersymmetry demands in this case that the 8-dimensional compact space be a Calabi-Yau 4-fold [1]3]. The warp factor in this background satisfies the equation (coming from the equation of motion for the 3 -form potential)

$$
\tilde{\nabla}^{2} e^{3 \phi / 2}=\tilde{\star}_{8}\left(X_{8}-\frac{1}{2} G \wedge G\right)-\sum_{j} \delta^{8}\left(y-y_{j}\right),
$$

where we must include (self-dual) 4-form flux $G$, and a topological term $X_{8}(R)=$ $\frac{1}{8 \cdot 4 !}\left(\operatorname{tr} R^{4}-\frac{1}{4}\left(\operatorname{tr} R^{2}\right)^{2}\right)$ to cancel the $C_{3}$ tadpole of the $M 2$-branes at the locations $y_{i}$,

$$
\frac{\chi_{C Y_{4}}}{24}=Q_{2}+\frac{1}{2} \int_{C Y_{4}} G \wedge G .
$$

We see that the shift ansatz for the volume modulus dependence in the warp factor (A.36) is quite natural since it is a zero mode of (A.37), even though it was derived in a very different way.

The case of $D=10$ reduced to $p+1=4$-dimensional Minkowski space has the metric (A.14) (renaming $u(x)=c(x))$ :

$$
d s_{10}^{2}=e^{2 A(y, c(x))} e^{2 \Omega[c(x)]}\left[\hat{\eta}_{\mu \nu}+2 \partial_{\mu} \partial_{\nu} c(x) K(y)\right] d x^{\mu} d x^{\nu}+e^{-2 A(y, c(x))} \tilde{g}_{m n}(y) d y^{m} d y^{n},
$$

with the shift ansatz (A.20) taking the form,

$$
e^{-4 A(y, u(x))}=e^{-4 A_{0}(y)}+c(x) .
$$

Again, the warped product structure of the metric (A.39) is identical to that of the corresponding extremal D3-brane solution [42]. Upon supersymmetric compactification to $(3+1)$-dimensional Minkowski space on D3-branes, again the warped product structure of the metric also takes this form, and supersymmetry demands in this case that the 6-dimensional compact space be a CalabiYau 3-fold [2,5]. The warp factor in this background satisfies the equation (coming from the equation of motion for the RR 4-form) [5]:

$$
-\tilde{\nabla}^{2} e^{-4 A}=\frac{\left|\widetilde{G}_{3}\right|^{2}}{12 \operatorname{Im} \tau}+2 \kappa_{10}^{2} T_{3} \rho_{3}^{\text {loc }},
$$

where we must include (imaginary self-dual) $G_{3}$ flux and orientifold $O 3$-planes to cancel the tadpole from the D3-brane charges,

$$
\int_{\mathcal{M}_{6}} H_{3} \wedge F_{3}+Q_{3}^{\mathrm{loc}}=0
$$

Again, we see that the shift form of the volume modulus dependence of the warp factor (A.40) is a natural zero mode of the background (A.41). The metric (A.39) is just the volume modulus deformation of the GKP background found in [12]. 
The final "special" case of $D=11$ reduced to $p+1=6$-dimensional Minkowksi space has the metric (A.14),

$$
d s_{11}^{2}=e^{2 A(y, u(x))} e^{2 \Omega(u(x))}\left[\hat{\eta}_{\mu \nu}+2 e^{2 \Omega} \partial_{\mu} \partial_{\nu} u(x) K(y)\right] d x^{\mu} d x^{\nu}+e^{-4 A(y, u(x))} \tilde{g}_{m n}(y) d y^{m} d y^{n}
$$

with the shift ansatz (A.20) taking the form

$$
e^{-6 A(y, u(x))}=e^{-6 A_{0}(y)}+u(x) .
$$

As expected, the warped product structure of (A.43) is identical to the supergravity solution of the extremal $M 5$-brane [49]. Upon compactification to $(5+1)$-dimensional Minkowski space on spacetime filling M5-branes, the warped product structure also takes this form [50], while low energy supersymmetry requires the internal space to be the orientifolds $T^{5} / \mathbb{Z}_{2}$ or $K 3 \times S^{1} / \mathbb{Z}_{2}$ (and their orbifolds) [51, 52. The warp factor satisfies the equation (coming from the Bianchi identity for the 4-form field strength),

$$
-\tilde{\nabla}^{2} e^{-6 A}=\sum_{i} q_{i} \delta^{5}\left(y-y_{i}\right)
$$

for $M 5$-branes $q_{i}=1$. In order to satisfy the tadpole constraint coming from (A.45) we must have additional sources of negative $M 5$-brane charge; in contrast to the $M 2$-brane case, flux cannot carry M5-brane charge, and so cannot be used to cancel this tadpole. Fortunately, as discussed in [52], the twisted sector fields at the fixed points of the orbifold action of the orientifold carry negative charge $q_{i}=-1 / 2$ (in just such a way that they are free of gravitational anomalies), so the tadpole condition coming from (A.45) can be satisfied. Again, we see that the shift form of the volume modulus in the warp factor (A.44) is a natural zero mode of the background (A.45).

It is perhaps remarkable that our simple analysis of a warped volume modulus, without any explicit reference to the form of additional matter, has led quite naturally to the $D 3-, M 2-$, and M5-brane backgrounds.

\section{References}

[1] K. Becker and M. Becker, "M-Theory on Eight-Manifolds," Nucl. Phys. B477 (1996) 155-167, hep-th/9605053.

[2] K. Dasgupta, G. Rajesh, and S. Sethi, "M theory, orientifolds and G-flux," JHEP 08 (1999) 023, hep-th/9908088.

[3] B. R. Greene, K. Schalm, and G. Shiu, "Warped compactifications in M and F theory," Nucl. Phys. B584 (2000) 480-508, hep-th/0004103.

[4] S. Kachru, R. Kallosh, A. Linde, and S. P. Trivedi, "De Sitter vacua in string theory," Phys. Rev. D68 (2003) 046005, hep-th/0301240.

[5] S. B. Giddings, S. Kachru, and J. Polchinski, "Hierarchies from fluxes in string compactifications," Phys. Rev. D66 (2002) 106006, hep-th/0105097.

\footnotetext{
${ }^{10}$ Strictly speaking an orientifold of M-theory is not well-defined, since there is no worldsheet theory to construct an orientifold with respect to. In practice, orientifolds in M-theory are defined by lifting the orientifold action in II $A$ to the M-theory fields, see [51,52] for some discussion.
} 
[6] A. R. Frey and J. Polchinski, "N = 3 warped compactifications," Phys. Rev. D65 (2002) 126009, hep-th/0201029.

[7] A. R. Frey, "Warped strings: Self-dual flux and contemporary compactifications," hep-th/0308156.

[8] M. R. Douglas, "Effective potential and warp factor dynamics," JHEP 03 (2010) 071, 0911.3378.

[9] M. R. Douglas and R. Kallosh, "Compactification on negatively curved manifolds," 1001.4008.

[10] S. B. Giddings and A. Maharana, "Dynamics of warped compactifications and the shape of the warped landscape," Phys. Rev. D73 (2006) 126003, hep-th/0507158.

[11] G. Shiu, G. Torroba, B. Underwood, and M. R. Douglas, "Dynamics of Warped Flux Compactifications," JHEP 06 (2008) 024, 0803.3068.

[12] A. R. Frey, G. Torroba, B. Underwood, and M. R. Douglas, "The Universal Kaehler Modulus in Warped Compactifications," JHEP 01 (2009) 036, 0810.5768.

[13] C. van de Bruck, M. Dorca, R. H. Brandenberger, and A. Lukas, "Cosmological perturbations in brane-world theories: Formalism," Phys. Rev. D62 (2000) 123515, hep-th/0005032.

[14] C. Csaki, M. L. Graesser, and G. D. Kribs, "Radion dynamics and electroweak physics," Phys. Rev. D63 (2001) 065002, hep-th/0008151.

[15] T. Tanaka and X. Montes, "Gravity in the brane-world for two-branes model with stabilized modulus," Nucl. Phys. B582 (2000) 259-276, hep-th/0001092.

[16] Z. Kakushadze and P. Langfelder, "Gravitational Higgs mechanism," Mod.Phys.Lett. A15 (2000) 2265-2280, hep-th/0011245.

[17] R. Rabadan and F. Zamora, "Dilaton tadpoles and D-brane interactions in compact spaces," JHEP 12 (2002) 052, hep-th/0207178.

[18] C. R. Contaldi, L. Kofman, and M. Peloso, "Gravitational instability of de Sitter compactifications," JCAP 0408 (2004) 007, hep-th/0403270.

[19] O. DeWolfe and S. B. Giddings, "Scales and hierarchies in warped compactifications and brane worlds," Phys. Rev. D67 (2003) 066008, hep-th/0208123.

[20] S. P. de Alwis, "On potentials from fluxes," Phys. Rev. D68 (2003) 126001, hep-th/0307084.

[21] A. Buchel, "On effective action of string theory flux compactifications," Phys. Rev. D69 (2004) 106004, hep-th/0312076.

[22] S. P. de Alwis, "Brane worlds in 5D and warped compactifications in IIB," Phys. Lett. B603 (2004) 230-238, hep-th/0407126.

[23] C. P. Burgess et al., "Warped supersymmetry breaking," JHEP 04 (2008) 053, hep-th/0610255. 
[24] M. R. Douglas, J. Shelton, and G. Torroba, "Warping and supersymmetry breaking," 0704.4001.

[25] M. R. Douglas and G. Torroba, "Kinetic terms in warped compactifications," JHEP 05 (2009) 013, 0805.3700.

[26] A. R. Frey and A. Maharana, "Warped spectroscopy: Localization of frozen bulk modes," JHEP 08 (2006) 021, hep-th/0603233.

[27] P. Koerber and L. Martucci, "From ten to four and back again: how to generalize the geometry," JHEP 08 (2007) 059, 0707.1038.

[28] L. Martucci, "On moduli and effective theory of $\mathrm{N}=1$ warped flux compactifications," JHEP 05 (2009) 027, 0902.4031.

[29] H.-Y. Chen, Y. Nakayama, and G. Shiu, "On D3-brane Dynamics at Strong Warping," Int. J. Mod. Phys. A25 (2010) 2493-2513, 0905.4463.

[30] F. Marchesano, P. McGuirk, and G. Shiu, "Open String Wavefunctions in Warped Compactifications," JHEP 04 (2009) 095, 0812.2247.

[31] P. Creminelli, M. A. Luty, A. Nicolis, and L. Senatore, "Starting the Universe: Stable Violation of the Null Energy Condition and Non-standard Cosmologies," JHEP 0612 (2006) 080, hep-th/0606090.

[32] C. Cheung, P. Creminelli, A. Fitzpatrick, J. Kaplan, and L. Senatore, "The Effective Field Theory of Inflation," JHEP 0803 (2008) 014, 0709.0293.

[33] R. M. Wald, General Relativity. University of Chicago Press, 1984.

[34] J. M. Bardeen, "Gauge Invariant Cosmological Perturbations," Phys. Rev. D22 (1980) 1882-1905.

[35] V. F. Mukhanov, H. A. Feldman, and R. H. Brandenberger, "Theory of cosmological perturbations. Part 1. Classical perturbations. Part 2. Quantum theory of perturbations. Part 3. Extensions," Phys. Rept. 215 (1992) 203-333.

[36] L. Randall and R. Sundrum, "A large mass hierarchy from a small extra dimension," Phys. Rev. Lett. 83 (1999) 3370-3373, hep-ph/9905221.

[37] L. Randall and R. Sundrum, "An alternative to compactification," Phys. Rev. Lett. 83 (1999) 4690-4693, hep-th/9906064.

[38] W. D. Goldberger and M. B. Wise, "Modulus stabilization with bulk fields," Phys. Rev. Lett. 83 (1999) 4922-4925, hep-ph/9907447.

[39] S. Aybat and D. P. George, "Stability of Scalar Fields in Warped Extra Dimensions," JHEP 1009 (2010) 010, 1006.2827.

[40] D. P. George, "Survival of scalar zero modes in warped extra dimensions," 1102.0564.

[41] P. K. Townsend, "Supersymmetric Extended Solitons," Phys. Lett. B202 (1988) 53. 
[42] G. T. Horowitz and A. Strominger, "Black strings and P-branes," Nucl. Phys. B360 (1991) 197-209.

[43] H. Lu, C. N. Pope, E. Sezgin, and K. S. Stelle, "Stainless super p-branes," Nucl. Phys. B456 (1995) 669-698, hep-th/9508042.

[44] J. Blaback, U. Danielsson, D. Junghans, T. Van Riet, T. Wrase, and M. Zagermann, "Smeared versus localised sources in flux compactifications," (2010) 1009.1877.

[45] K.-i. Maeda, M. Minamitsuji, N. Ohta, and K. Uzawa, "Dynamical p-branes with a cosmological constant," Phys. Rev. D82 (2010) 046007, 1006.2306.

[46] D. Lust, F. Marchesano, L. Martucci, and D. Tsimpis, "Generalized non-supersymmetric flux vacua," JHEP 0811 (2008) 021, 0807.4540, * Temporary entry *.

[47] D. Roest, "M-theory and gauged supergravities," Fortsch. Phys. 53 (2005) 119-230, hep-th/0408175.

[48] M. J. Duff and K. S. Stelle, "Multi-membrane solutions of D = 11 supergravity," Phys. Lett. B253 (1991) 113-118.

[49] R. Guven, "Black p-brane solutions of D=11 supergravity theory," Phys. Lett. B276 (1992) $49-55$.

[50] E. Witten, "Strong Coupling Expansion Of Calabi-Yau Compactification," Nucl. Phys. B471 (1996) 135-158, hep-th/9602070.

[51] K. Dasgupta and S. Mukhi, "Orbifolds of M-theory," Nucl. Phys. B465 (1996) 399-412, hep-th/9512196.

[52] E. Witten, "Five-branes and M-theory on an orbifold," Nucl. Phys. B463 (1996) 383-397, hep-th/9512219. 